\title{
AN ADAPTIVE PERFECTLY MATCHED LAYER TECHNIQUE FOR 3-D TIME-HARMONIC ELECTROMAGNETIC SCATTERING PROBLEMS
}

\author{
JUNQING CHEN AND ZHIMING CHEN
}

\begin{abstract}
An adaptive perfectly matched layer (PML) technique for solving the time harmonic electromagnetic scattering problems is developed. The PML parameters such as the thickness of the layer and the fictitious medium property are determined through sharp a posteriori error estimates. Combined with the adaptive finite element method, the adaptive PML technique provides a complete numerical strategy to solve the scattering problem in the framework of FEM which produces automatically a coarse mesh size away from the fixed domain and thus makes the total computational costs insensitive to the thickness of the PML absorbing layer. Numerical experiments are included to illustrate the competitive behavior of the proposed adaptive method.
\end{abstract}

\section{INTRODUCTION}

We propose and study an adaptive perfectly matched layer (PML) technique for solving the time harmonic electromagnetic scattering problem with the perfectly conducting boundary condition

$$
\begin{aligned}
& \nabla \times \nabla \times \mathbf{E}-k^{2} \mathbf{E}=0 \quad \text { in } \mathbb{R}^{3} \backslash \bar{D}, \\
& \mathbf{n} \times \mathbf{E}=\mathbf{g} \quad \text { on } \Gamma_{D}, \\
& |\mathbf{x}|[(\nabla \times \mathbf{E}) \times \hat{\mathbf{x}}-1 k \mathbf{E}] \rightarrow 0 \quad \text { as }|\mathbf{x}| \rightarrow \infty .
\end{aligned}
$$

Here $D \subset \mathbb{R}^{3}$ is a bounded domain with Lipschitz polyhedral boundary $\Gamma_{D}, \mathbf{E}$ is the electric field, $\mathbf{g}$ is determined by the incoming wave, $\hat{\mathbf{x}}=\mathbf{x} /|\mathbf{x}|$, and $\mathbf{n}$ is the unit outer normal to $\Gamma_{D}$. We assume the wave number $k \in \mathbb{R}$ is a constant. We remark that the results in this paper can easily be extended to solve the scattering problems with other boundary conditions such as Neumann or the impedance boundary condition on $\Gamma_{D}$, or to solve the electromagentic wave propagation through inhomogeneous media with a variable wave number $k^{2}(\mathbf{x})$ inside some bounded domain.

Since the work of Bérénger [5] which proposed a PML technique for solving the time dependent Maxwell equations, various constructions of PML absorbing layers have been proposed and studied in the literature (cf. e.g. Turkel and Yefet 24, Teixeira and Chew 23. for the reviews). Under the assumption that the exterior solution is composed of outgoing waves only, the basic idea of the PML technique is to surround the computational domain by a layer of finite thickness with a specially

Received by the editor March 6, 2006 and, in revised form, February 25, 2007.

2000 Mathematics Subject Classification. Primary 65N30, 65N50, 78A25.

This work was supported in part by China NSF under the grant 10428105 and by the National Basic Research Project under the grant 2005CB321701. 
designed model medium that would either slow down or attenuate all the waves that propagate from inside the computational domain.

The convergence of the PML method is studied in Lassas and Somersalo [16, Hohage, Schmidt and Zschiedrich [15] for the acoustic scattering problems and in Bao and $\mathrm{Wu}$ [3], Bramble and Pasciak 7] for the electromagnetic scattering problems. It is proved in [16, 15, 7] that the PML solution converges exponentially to the solution of the original scattering problem as the thickness of the PML layer tends to be infinite. We remark that in practical applications involving PML techniques, one cannot afford to use a very thick PML layer if uniform meshes are used because it requires excessive grid points and hence more computer time and more storage. On the other hand, a thin PML layer requires a rapid variation of the artificial material property which deteriorates the accuracy if too coarse a mesh is used in the PML layer.

The adaptive PML technique was first proposed in Chen and Wu [10] for a scattering problem by periodic structures (the grating problem) and in Chen and Liu 8 for the acoustic scattering problem in which one uses the a posteriori error estimate to determine the PML parameters. Combined with the adaptive finite element method, the adaptive PML technique provides a complete numerical strategy to solve the scattering problems in the framework of a finite element which automatically produces a coarse mesh size away from the fixed domain and thus makes the total computational costs insensitive to the thickness of the PML absorbing layer.

A posteriori error estimates are computable quantities in terms of the discrete solution and data that measure the actual discrete errors without the knowledge of exact solutions. The adaptive finite element method based on a posteriori error estimates provides a systematic way to achieve the optimal computational complexity by refining the mesh according to the local a posteriori error estimator on the elements. A posteriori error estimates for the Nédélec $\mathbf{H}(\mathbf{c u r l})$-conforming edge elements are obtained in Monk [17] for Maxwell scattering problems, in Beck, Hiptmair, Hoppe and Wohlmuth 4 for eddy current problems, and in Chen, Wang and Zheng [9] for Maxwell cavity problems. The restriction in [17, 4] that the domain should be convex or have smooth boundary in order to ensure the regularity of the function in the Helmholtz decomposition is removed in [9] by using the Birman-Solomyak decomposition [6].

In this paper we extend the idea of using a posteriori error estimates to determine the PML parameters for solving the electromagnetic scattering problem (1.1)-(1.3). Our technique to prove the PML convergence is different from the methods used in [7, 3. The key ingredient in our analysis is the following uniform estimate for the Hankel function $H_{\nu}^{(1)}(z)$ in [8]:

$$
\left|H_{\nu}^{(1)}(z)\right| \leq e^{-\operatorname{Im}(z)\left(1-\frac{\Theta^{2}}{|z|^{2}}\right)^{1 / 2}}\left|H_{\nu}^{(1)}(\Theta)\right|
$$

for any $z \in \mathbb{C}$ such that $\operatorname{Im}(z) \geq 0, \operatorname{Re}(z) \geq 0$ and $\Theta \in \mathbb{R}$ satisfying $0<\Theta<|z|$. This estimate, together with a uniform estimate of $\delta_{n}(z)=1+z \frac{h_{n}^{(1)^{\prime}}(z)}{h_{n}^{(1)}(z)}$ for $z>0$ due to Nédeléc [20, leads to the following crucial exponentially decaying property of the PML extension $\mathbb{E}(\lambda)$ (see (2.9) below for the definition):

$$
\|\hat{\mathbf{x}} \times \mathbb{E}(\lambda)\|_{\mathbf{H}^{-1 / 2}\left(\operatorname{Div} ; \Gamma_{\rho}\right)} \leq C(1+k R) e^{-\operatorname{Im}(k \tilde{\rho})\left(1-\frac{R^{2}}{|\tilde{\rho}|^{2}}\right)^{1 / 2}}\|\lambda\|_{\mathbf{H}^{-1 / 2}\left(\operatorname{Div} ; \Gamma_{\mathbf{R}}\right)} .
$$


Based on this estimate and the stability estimates for the PML equation in the PML layer, the following error estimate between the solution $\mathbf{E}$ of the scattering problem and the solution $\hat{\mathbf{E}}$ of the PML problem is proved (see Theorem 3.1 below):

$$
\|\mathbf{E}-\hat{\mathbf{E}}\|_{\mathbf{H}\left(\operatorname{curl} ; \Omega_{R}\right)} \leq C \hat{C}^{-1}(1+k R)^{3}\left|\alpha_{0}\right|^{3} e^{-\operatorname{Im}(k \tilde{\rho})\left(1-\frac{R^{2}}{|\hat{\rho}|^{2}}\right)^{1 / 2}}\|\hat{\mathbf{x}} \times \hat{\mathbf{E}}\|_{\mathbf{H}^{-1 / 2}\left(\operatorname{Div} ; \Gamma_{\mathrm{R}}\right)} .
$$

Let $\mathbf{E}_{h}$ be the finite element solution of the PML problem. The following a posteriori error estimate which is the basis of our adaptive PML method is derived (see Theorem 4.1 below):

$$
\begin{aligned}
& \left\|\mathbf{E}-\mathbf{E}_{h}\right\|_{\mathbf{H}\left(\mathbf{c u r l} ; \Omega_{R}\right)} \\
\leq & C\left\|\mathbf{g}-\mathbf{g}_{h}\right\|_{\mathbf{H}^{-1 / 2}\left(\operatorname{Div} ; \Gamma_{\mathrm{D}}\right)}+C \hat{C}^{-1}\left|\alpha_{0}\right|^{3}(1+k R)^{3} R^{1 / 2}\left(\sum_{K \in \mathcal{M}_{h}} \eta_{K}^{2}\right)^{1 / 2} \\
& +C \hat{C}^{-1}\left|\alpha_{0}\right|^{3}(1+k R)^{3} e^{-\operatorname{Im}(k \tilde{\rho})\left(1-\frac{R^{2}}{|\tilde{\rho}|^{2}}\right)^{1 / 2}}\left\|\hat{\mathbf{x}} \times \mathbf{E}_{h}\right\|_{\mathbf{H}^{-1 / 2}\left(\operatorname{Div} ; \Gamma_{\mathrm{R}}\right)} .
\end{aligned}
$$

Here $\mathbf{g}_{h}$ is the finite element approximation of the boundary value $\mathbf{g}$ and $\eta_{K}$ is the a posteriori error indicator on the element $K$ of residual type.

The layout of the paper is as follows. In section 2 we recall the PML formulation for (1.1)-(1.3). In section 3 we study the existence, uniqueness and convergence of the PML formulation. In section 4 we introduce the finite element discretization. In section 5 we derive the sharp a posteriori error estimate which lays down the basis of the combined adaptive PML and finite element methods. In section 6 we discuss the implementation of the adaptive method and present several numerical examples to illustrate the competitive behavior of the method.

\section{The PML EQUATION}

Let $D$ be contained in the interior of the ball $B_{R}=\left\{\mathbf{x} \in \mathbb{R}^{3},|\mathbf{x}|<R\right\}$ with boundary $\Gamma_{R}$. We first recall the series solution of the scattering problem (1.1)(1.3) outside the ball $B_{R}$ by following the development in Monk [18. Let $Y_{n}^{m}(\hat{\mathbf{x}})$, $m=-n, \ldots, n, n=1,2, \ldots$, be the spherical harmonics which satisfy

$$
\Delta_{\partial B_{1}} Y_{n}^{m}(\hat{\mathbf{x}})+n(n+1) Y_{n}^{m}(\hat{\mathbf{x}})=0 \quad \text { on } \partial B_{1},
$$

where $\Delta_{\partial B_{1}}=\frac{1}{\sin \theta} \frac{\partial}{\partial \theta}\left(\sin \theta \frac{\partial}{\partial \theta}\right)+\frac{1}{\sin ^{2} \theta} \frac{\partial^{2}}{\partial \phi^{2}}$ is the Laplace-Beltrami operator for the surface of the unit sphere $\partial B_{1}$. The set of all spherical harmonics $\left\{Y_{n}^{m}(\hat{\mathbf{x}}): m=\right.$ $-n, \ldots, n, n=1,2, \ldots\}$ forms a complete orthonormal basis of $L^{2}\left(\partial B_{1}\right)$.

Denote the vector spherical harmonics

$$
\mathbf{U}_{n}^{m}=\frac{1}{\sqrt{n(n+1)}} \nabla_{\partial B_{1}} Y_{n}^{m}, \quad \mathbf{V}_{n}^{m}=\hat{\mathbf{x}} \times \mathbf{U}_{n}^{m},
$$

where $\nabla_{\partial B_{1}} Y_{n}^{m}=\frac{\partial Y_{n}^{m}}{\partial \theta} \mathbf{e}_{\theta}+\frac{1}{\sin \theta} \frac{\partial Y_{n}^{m}}{\partial \phi} \mathbf{e}_{\phi}$, and $\left\{\mathbf{e}_{r}, \mathbf{e}_{\theta}, \mathbf{e}_{\phi}\right\}$ are the unit vectors of the spherical coordinates. The set of all vector spherical harmonics $\left\{\mathbf{U}_{n}^{m}, \mathbf{V}_{n}^{m}: m=\right.$ $-n, \ldots, n, n=1,2, \ldots\}$ forms a complete orthonormal basis of $\mathbf{L}_{t}^{2}\left(\partial B_{1}\right)=\{\mathbf{u} \in$ $L^{2}\left(\partial B_{1}\right)^{3}: \mathbf{u} \cdot \hat{\mathbf{x}}=0$ on $\left.\partial B_{1}\right\}$.

For any $\boldsymbol{\Phi} \in \mathbf{H}\left(\mathbf{c u r l}, B_{R}\right), \hat{\mathbf{x}} \times\left.\boldsymbol{\Phi}\right|_{\Gamma_{R}}$ is in the trace space $\mathbf{H}^{-1 / 2}\left(\right.$ Div; $\left.\Gamma_{\mathrm{R}}\right)$, whose norm, for any $\lambda=\sum_{n=1}^{\infty} \sum_{m=-n}^{n} a_{n m} \mathbf{U}_{n}^{m}+b_{n m} \mathbf{V}_{n}^{m}$, is defined by

$$
\|\lambda\|_{\mathbf{H}^{-1 / 2}\left(\mathrm{Div} ; \Gamma_{\mathrm{R}}\right)}^{2}=\sum_{n=1}^{\infty} \sum_{m=-n}^{n} \sqrt{n(n+1)}\left|a_{n m}\right|^{2}+\frac{1}{\sqrt{n(n+1)}}\left|b_{n m}\right|^{2} .
$$


It is also known that for $\boldsymbol{\Phi} \in \mathbf{H}\left(\mathbf{c u r l} ; B_{R}\right)$, the tangential component $(\hat{\mathbf{x}} \times \mathbf{\Phi}) \times\left.\hat{\mathbf{x}}\right|_{\Gamma_{R}}$ belongs to $\mathbf{H}^{-1 / 2}\left(\mathrm{Curl} ; \Gamma_{\mathrm{R}}\right)$ which is the dual space of $\mathbf{H}^{-1 / 2}\left(\operatorname{Div} ; \Gamma_{\mathrm{R}}\right)$ with respect to the scalar product in $\mathbf{L}_{t}^{2}\left(\Gamma_{R}\right)$ [20, Theorem 5.4.2, Lemma 5.3.1]. In the following we will always denote by $\langle\cdot, \cdot\rangle_{\Gamma_{R}}$ the duality pairing between $\mathbf{H}^{-1 / 2}\left(\right.$ Div; $\left.\Gamma_{\mathrm{R}}\right)$ and $\mathbf{H}^{-1 / 2}\left(\mathrm{Curl} ; \Gamma_{\mathrm{R}}\right)$.

Let $h_{n}^{(1)}(z)$ be the spherical Hankel function of the first kind of order $n$. We introduce the vector wave functions

$$
\mathbf{M}_{n}^{m}(r, \hat{\mathbf{x}})=\nabla \times\left\{\mathbf{x} h_{n}^{(1)}(k r) Y_{n}^{m}(\hat{\mathbf{x}})\right\}, \quad \mathbf{N}_{n}^{m}(r, \hat{\mathbf{x}})=\frac{1}{1 k} \nabla \times \mathbf{M}_{n}^{m}(r, \hat{\mathbf{x}}),
$$

which are the radiation solutions of the Maxwell equation (1.1) in $\mathbb{R}^{3} \backslash\{0\}$. In the domain $\mathbb{R}^{3} \backslash \bar{B}_{R}$, the solution $\mathbf{E}$ of (1.1)-(1.3) can be written as

$$
\mathbf{E}(r, \hat{\mathbf{x}})=\sum_{n=1}^{\infty} \sum_{m=-n}^{n} \frac{a_{n m} \mathbf{M}_{n}^{m}(r, \hat{\mathbf{x}})}{h_{n}^{(1)}(k R) \sqrt{n(n+1)}}+\frac{1 k R b_{n m} \mathbf{N}_{n}^{m}(r, \hat{\mathbf{x}})}{z_{n}^{(1)}(k R) \sqrt{n(n+1)}}
$$

where $z_{n}^{(1)}(k R)=h_{n}^{(1)}(k R)+k R h_{n}^{(1) \prime}(k R)$, and $a_{n m}, b_{n m}$ are determined by the trace of $\mathbf{E}$ on $\Gamma_{R}$ through $\hat{\mathbf{x}} \times\left.\mathbf{E}\right|_{\Gamma_{R}}=\sum_{n=1}^{\infty} \sum_{m=-n}^{n} a_{n m} \mathbf{U}_{n}^{m}+b_{n m} \mathbf{V}_{n}^{m}$. The series in (2.4) converges uniformly for $r>R$.

Now we turn to the introduction of the absorbing PML layer. We surround the domain $\Omega_{R}=B_{R} \backslash \bar{D}$ with a PML layer $\Omega^{\mathrm{PML}}=\left\{\mathbf{x} \in \mathbb{R}^{3}: R<|\mathbf{x}|<\rho\right\}$. Throughout the paper we assume $\rho \leq C R$ for some generic constant $C>0$. Let $\alpha(r)=1+1 \sigma(r)$ be the model medium property which satisfies

$$
\sigma \in C(\mathbb{R}), \quad \sigma \geq 0, \quad \text { and } \sigma=0 \text { for } r \leq R .
$$

Denote by $\tilde{r}$ the complex radius defined by

$$
\tilde{r}=\tilde{r}(r)= \begin{cases}r & \text { if } r \leq R, \\ \int_{0}^{r} \alpha(t) d t=r \beta(r) & \text { if } r \geq R .\end{cases}
$$

It is easy to check that the vector wave functions satisfy

$$
\begin{aligned}
\mathbf{M}_{n}^{m}(r, \hat{\mathbf{x}}) & =h_{n}^{(1)}(k r) \nabla_{\partial B_{1}} Y_{n}^{m}(\hat{\mathbf{x}}) \times \hat{\mathbf{x}} \\
\mathbf{N}_{n}^{m}(r, \hat{\mathbf{x}}) & =\frac{1}{1 k} \nabla \times \mathbf{M}_{n}^{m} \\
& =\frac{\sqrt{n(n+1)}}{1 k r} z_{n}^{(1)}(k r) \mathbf{U}_{n}^{m}(\hat{\mathbf{x}})+\frac{n(n+1)}{1 k r} h_{n}^{(1)}(k r) Y_{n}^{m}(\hat{\mathbf{x}}) \hat{\mathbf{x}}
\end{aligned}
$$

We introduce

$$
\begin{aligned}
\tilde{\mathbf{M}}_{n}^{m}(\tilde{r}, \hat{\mathbf{x}}) & =h_{n}^{(1)}(k \tilde{r}) \nabla_{\partial B_{1}} Y_{n}^{m}(\hat{\mathbf{x}}) \times \hat{\mathbf{x}} \\
\tilde{\mathbf{N}}_{n}^{m}(\tilde{r}, \hat{\mathbf{x}}) & =\frac{1}{1 k} \tilde{\nabla} \times \tilde{\mathbf{M}}_{n}^{m} \\
& =\frac{\sqrt{n(n+1)}}{1 k \tilde{r}} z_{n}^{(1)}(k \tilde{r}) \mathbf{U}_{n}^{m}(\hat{\mathbf{x}})+\frac{n(n+1)}{1 k \tilde{r}} h_{n}^{(1)}(k \tilde{r}) Y_{n}^{m}(\hat{\mathbf{x}}) \hat{\mathbf{x}}
\end{aligned}
$$


where $\tilde{\nabla} \times$ is the curl operator with respect to the complex spherical variables $(\tilde{r}, \theta, \phi)$, that is, for $\boldsymbol{\Phi}=\boldsymbol{\Phi}_{r} \mathbf{e}_{r}+\boldsymbol{\Phi}_{\theta} \mathbf{e}_{\theta}+\boldsymbol{\Phi}_{\phi} \mathbf{e}_{\phi}$,

$$
\begin{aligned}
\tilde{\nabla} \times \boldsymbol{\Phi}= & \frac{1}{\tilde{r} \sin \theta}\left(\frac{\partial}{\partial \theta}\left(\sin \theta \boldsymbol{\Phi}_{\phi}\right)-\frac{\partial \boldsymbol{\Phi}_{\theta}}{\partial \phi}\right) \mathbf{e}_{r} \\
& +\frac{1}{\tilde{r}}\left(\frac{1}{\sin \theta} \frac{\partial \boldsymbol{\Phi}_{r}}{\partial \phi}-\frac{\partial\left(\tilde{r} \boldsymbol{\Phi}_{\phi}\right)}{\partial \tilde{r}}\right) \mathbf{e}_{\theta} \\
& +\frac{1}{\tilde{r}}\left(\frac{\partial\left(\tilde{r} \boldsymbol{\Phi}_{\theta}\right)}{\partial \tilde{r}}-\frac{\partial \boldsymbol{\Phi}_{\phi}}{\partial \theta}\right) \mathbf{e}_{\phi} .
\end{aligned}
$$

It is easy to check that $\tilde{\nabla} \times \boldsymbol{\Phi}=A \nabla \times B \boldsymbol{\Phi}$, where $A=\operatorname{diag}\left(\beta^{-2}, \alpha^{-1} \beta^{-1}, \alpha^{-1} \beta^{-1}\right)$ and $B=\operatorname{diag}(\alpha, \beta, \beta)$ are $3 \times 3$ diagonal matrices.

We follow [18] to derive the PML equation. For any $\lambda=\sum_{n=1}^{\infty} \sum_{m=-n}^{n} a_{n m} \mathbf{U}_{n}^{m}+$ $b_{n m} \mathbf{V}_{n}^{m} \in \mathbf{H}^{-1 / 2}\left(\operatorname{Div} ; \Gamma_{\mathrm{R}}\right)$, let $\mathbb{E}(\lambda)(\tilde{r}, \hat{\mathbf{x}})$ be the PML extension given by

$$
\mathbb{E}(\lambda)(\tilde{r}, \hat{\mathbf{x}})=\sum_{n=1}^{\infty} \sum_{m=-n}^{n} \frac{a_{n m} \tilde{\mathbf{M}}_{n}^{m}(\tilde{r}, \hat{\mathbf{x}})}{h_{n}^{(1)}(k R) \sqrt{n(n+1)}}+\frac{1 k R b_{n m} \tilde{\mathbf{N}}_{n}^{m}(\tilde{r}, \hat{\mathbf{x}})}{z_{n}^{(1)}(k R) \sqrt{n(n+1)}}, \quad r>R
$$

For the solution $\mathbf{E}$ of the scattering problem (1.1)-(1.3), let $\tilde{\mathbf{E}}=\mathbb{E}\left(\hat{\mathbf{x}} \times\left.\mathbf{E}\right|_{\Gamma_{R}}\right)$ be the PML extension of $\hat{\mathbf{x}} \times\left.\mathbf{E}\right|_{\Gamma_{R}}$. Since $\tilde{r}=r$ on $\Gamma_{R}$, we know that $\hat{\mathbf{x}} \times \tilde{\mathbf{E}}=\hat{\mathbf{x}} \times \mathbf{E}$ on $\Gamma_{R}$. On the other hand, since $h_{n}^{(1)}(z) \sim \frac{1}{z} e^{1\left(z-\frac{1}{2} n \pi-\frac{1}{2} \pi\right)}$ asymptotically as $|z| \rightarrow \infty$, heuristically $\tilde{\mathbf{E}}(\tilde{r}, \hat{\mathbf{x}})$ will decay exponentially for large $r>R$. It is obvious that $\tilde{\mathbf{E}}$ satisfies

$$
\tilde{\nabla} \times \tilde{\nabla} \times \tilde{\mathbf{E}}-k^{2} \tilde{\mathbf{E}}=0 \quad \text { in } \quad \mathbb{R}^{3} \backslash \bar{B}_{R},
$$

which gives the desired PML equation in the spherical coordinates

$$
\nabla \times B(A \nabla \times B \tilde{\mathbf{E}})-k^{2} A^{-1} \tilde{\mathbf{E}}=0 \quad \text { in } \quad \mathbb{R}^{3} \backslash \bar{B}_{R} .
$$

The PML problem is then to find $\hat{\mathbf{E}}$, which approximates $\mathbf{E}$ in $\Omega_{R}$ and $B \tilde{\mathbf{E}}$ in $\Omega^{\mathrm{PML}}=B_{\rho} \backslash \bar{B}_{R}$, as the solution of the following system:

$$
\begin{aligned}
& \nabla \times B A(\nabla \times \hat{\mathbf{E}})-k^{2}(B A)^{-1} \hat{\mathbf{E}}=0 \quad \text { in } \Omega_{\rho}=B_{\rho} \backslash \bar{D}, \\
& \mathbf{n} \times \hat{\mathbf{E}}=\mathbf{g} \text { on } \Gamma_{D}, \quad \hat{\mathbf{x}} \times \hat{\mathbf{E}}=0 \text { on } \Gamma_{\rho} .
\end{aligned}
$$

The well-posedness of the PML problem (2.10)-(2.11) and the convergence of its solution to the solution of the original problem (1.1)-1.3 will be studied in the next section.

In the remainder of this section we introduce the equivalent variational form of the scattering problem (1.1)-(1.3) and the PML problem (2.10)-(2.11) on the bounded domain $\Omega_{R}=B_{R} \backslash \bar{D}$ using the Calderon operators.

Given a tangential vector $\lambda$ on $\Gamma_{R}$, the Calderon operator $G_{e}: \mathbf{H}^{-1 / 2}\left(\operatorname{Div} ; \Gamma_{\mathrm{R}}\right) \rightarrow$ $\mathbf{H}^{-1 / 2}\left(\operatorname{Div} ; \Gamma_{\mathrm{R}}\right)$ is the Dirichlet to Neumann operator defined by

$$
G_{e}(\lambda)=\frac{1}{1 k} \hat{\mathbf{x}} \times\left(\nabla \times \mathbf{E}^{s}\right),
$$

where $\mathbf{E}^{s}$ satisfies

$$
\begin{aligned}
& \nabla \times \nabla \times \mathbf{E}^{s}-k^{2} \mathbf{E}^{s}=0 \quad \text { in } \mathbb{R}^{3} \backslash \bar{B}_{R} \\
& \hat{\mathbf{x}} \times \mathbf{E}^{s}=\lambda \text { on } \Gamma_{R}, \\
& |\mathbf{x}|\left[\left(\nabla \times \mathbf{E}^{s}\right) \times \hat{\mathbf{x}}-1 k \mathbf{E}^{s}\right] \rightarrow 0 \text { as }|\mathbf{x}| \rightarrow \infty
\end{aligned}
$$


Let $\lambda=\sum_{n=1}^{\infty} \sum_{m=-n}^{n} a_{n m} \mathbf{U}_{n}^{m}+b_{n m} \mathbf{V}_{n}^{m}$; the function $\mathbf{E}^{s}$ is given as in (2.4)

$$
\mathbf{E}^{s}(r, \hat{\mathbf{x}})=\sum_{n=1}^{\infty} \sum_{m=-n}^{n} \frac{a_{n m} \mathbf{M}_{n}^{m}(r, \hat{\mathbf{x}})}{h_{n}^{(1)}(k R) \sqrt{n(n+1)}}+\frac{1 k R b_{n m} \mathbf{N}_{n}^{m}(r, \hat{\mathbf{x}})}{z_{n}^{(1)}(k R) \sqrt{n(n+1)}}, \quad r>R .
$$

Since $\frac{1}{1 k} \nabla \times \mathbf{M}_{n}^{m}=\mathbf{N}_{m}^{n},-\frac{1}{1 k} \nabla \times \mathbf{N}_{n}^{m}=\mathbf{M}_{n}^{m}$, we have

$$
\frac{1}{1 k} \nabla \times \mathbf{E}^{s}=\sum_{n=1}^{\infty} \sum_{m=-n}^{n} \frac{a_{m n} \mathbf{N}_{n}^{m}}{h_{n}^{(1)}(k R) \sqrt{n(n+1)}}-\frac{1 k R b_{m n} \mathbf{M}_{n}^{m}}{z_{n}^{(1)}(k R) \sqrt{n(n+1)}} .
$$

Thus, by (2.5)-(2.6),

$$
G_{e}(\lambda)=\sum_{n=1}^{\infty} \sum_{m=-n}^{n} \frac{-1 k R b_{n m} h_{n}^{(1)}(k R)}{z_{n}^{(1)}(k R)} \mathbf{U}_{n}^{m}(\hat{\mathbf{x}})+\frac{a_{n m} z_{n}^{(1)}(k R)}{1 k R h_{n}^{(1)}(k R)} \mathbf{V}_{n}^{m}(\hat{\mathbf{x}}) .
$$

Let $a: \mathbf{H}\left(\mathbf{c u r l}, \Omega_{R}\right) \times \mathbf{H}\left(\mathbf{c u r l}, \Omega_{R}\right) \rightarrow \mathbb{C}$ be the sesquilinear form

$$
a(\mathbf{E}, \mathbf{\Phi})=\int_{\Omega_{R}}\left(\nabla \times \mathbf{E} \cdot \nabla \times \overline{\mathbf{\Phi}}-k^{2} \mathbf{E} \cdot \overline{\mathbf{\Phi}}\right) d \mathbf{x}+1 k\left\langle G_{e}(\hat{\mathbf{x}} \times \mathbf{E}),(\hat{\mathbf{x}} \times \mathbf{\Phi}) \times \hat{\mathbf{x}}\right\rangle_{\Gamma_{R}} .
$$

The scattering problem (1.1)-(1.3) is equivalent to the following weak formulation: Given $\mathbf{g} \in \mathbf{H}^{-1 / 2}\left(\operatorname{Div} ; \Gamma_{\mathrm{D}}\right)$, find $\mathbf{E} \in \mathbf{H}\left(\mathbf{c u r l} ; \Omega_{R}\right)$ such that $\mathbf{n} \times \mathbf{E}=\mathbf{g}$ on $\Gamma_{D}$, and

$$
a(\mathbf{E}, \boldsymbol{\Phi})=0, \quad \forall \boldsymbol{\Phi} \in \mathbf{H}_{D}\left(\operatorname{curl} ; \Omega_{R}\right),
$$

where $\mathbf{H}_{D}\left(\mathbf{c u r l} ; \Omega_{R}\right)=\left\{\mathbf{v} \in \mathbf{H}\left(\mathbf{c u r l} ; \Omega_{R}\right): \mathbf{n} \times \mathbf{v}=0\right.$ on $\left.\Gamma_{D}\right\}$.

The existence of a unique solution of the variational problem (2.13) is known [18, 20. Then the general theory in Babuška and Aziz [1] implies that there exists a constant $\mu>0$ such that the following inf-sup condition holds:

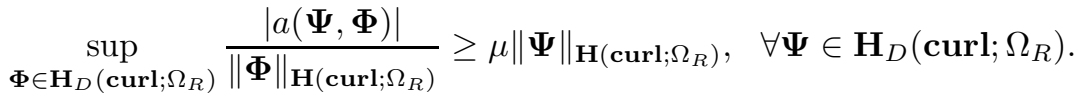

To study the convergence of the PML problem (2.10)-2.11), we need to reformulate it in the bounded domain $\Omega_{R}$ by imposing the boundary condition

$$
\hat{\mathbf{x}} \times\left.(\nabla \times \hat{\mathbf{E}})\right|_{\Gamma_{R}}=1 k \hat{G}_{e}\left(\hat{\mathbf{x}} \times\left.\hat{\mathbf{E}}\right|_{\Gamma_{R}}\right),
$$

where the approximate Calderon operator $\hat{G}_{e}: \mathbf{H}^{-1 / 2}\left(\operatorname{Div} ; \Gamma_{\mathrm{R}}\right) \rightarrow \mathbf{H}^{-1 / 2}\left(\mathrm{Div} ; \Gamma_{\mathrm{R}}\right)$ is defined as

$$
\hat{G}_{e}(\lambda):=\frac{1}{{ }_{1} k} \hat{\mathbf{x}} \times(\nabla \times \boldsymbol{\Psi}),
$$

with $\Psi$ satisfying

$$
\begin{aligned}
& \nabla \times B A(\nabla \times \boldsymbol{\Psi})-k^{2}(B A)^{-1} \boldsymbol{\Psi}=0 \quad \text { in } \Omega^{\mathrm{PML}}, \\
& \hat{\mathbf{x}} \times \boldsymbol{\Psi}=\lambda \text { on } \Gamma_{R}, \quad \hat{\mathbf{x}} \times \boldsymbol{\Psi}=0 \text { on } \Gamma_{\rho} .
\end{aligned}
$$

That the approximate Calderon operator $\hat{G}_{e}$ is well-defined will be studied in the next section. Based on the operator $\hat{G}_{e}$, let $\hat{a}: \mathbf{H}\left(\operatorname{curl} ; \Omega_{R}\right) \times \mathbf{H}\left(\operatorname{curl} ; \Omega_{R}\right) \rightarrow \mathbb{C}$ be the sesquilinear form

$$
\hat{a}(\hat{\mathbf{E}}, \mathbf{\Phi})=\int_{\Omega_{R}}\left(\nabla \times \hat{\mathbf{E}} \cdot \nabla \times \overline{\mathbf{\Phi}}-k^{2} \hat{\mathbf{E}} \cdot \overline{\mathbf{\Phi}}\right) d \mathbf{x}+1 k\left\langle\hat{G}_{e}(\hat{\mathbf{x}} \times \hat{\mathbf{E}}),(\hat{\mathbf{x}} \times \mathbf{\Phi}) \times \hat{\mathbf{x}}\right\rangle_{\Gamma_{R}} .
$$

Then the weak formulation of (2.10)-(2.11) on the bounded domain $\Omega_{R}$ is: Given $\mathbf{g} \in \mathbf{H}^{-1 / 2}\left(\operatorname{Div} ; \Gamma_{\mathrm{D}}\right)$, find $\hat{\mathbf{E}} \in \mathbf{H}\left(\mathbf{c u r l} ; \Omega_{R}\right)$, such that $\mathbf{n} \times \hat{\mathbf{E}}=\mathbf{g}$ on $\Gamma_{D}$, and

$$
\hat{a}(\hat{\mathbf{E}}, \boldsymbol{\Phi})=0, \quad \forall \boldsymbol{\Phi} \in \mathbf{H}_{D}\left(\operatorname{curl} ; \Omega_{R}\right) .
$$




\section{Convergence of the PML problem}

We start by considering the Dirichlet problem of the PML equation in the layer

$$
\begin{aligned}
& \nabla \times B A \nabla \times \mathbf{v}-k^{2}(B A)^{-1} \mathbf{v}=0 \text { in } \Omega^{\mathrm{PML}}, \\
& \hat{\mathbf{x}} \times \mathbf{v}=0 \text { on } \Gamma_{R}, \quad \hat{\mathbf{x}} \times \mathbf{v}=\mathbf{q} \text { on } \Gamma_{\rho},
\end{aligned}
$$

where $\mathbf{q} \in \mathbf{H}^{-1 / 2}\left(\operatorname{Div} ; \Gamma_{\rho}\right)$. Introduce the sesquilinear form $c: \mathbf{H}\left(\mathbf{c u r l} ; \Omega^{\mathrm{PML}}\right) \times$ $\mathbf{H}\left(\operatorname{curl} ; \Omega^{\mathrm{PML}}\right) \rightarrow \mathbb{C}$ as

$$
c(\mathbf{v}, \mathbf{\Phi})=\int_{\Omega^{\mathrm{PML}}}\left(B A \nabla \times \mathbf{v} \cdot \nabla \times \overline{\mathbf{\Phi}}-k^{2}(B A)^{-1} \mathbf{v} \cdot \overline{\mathbf{\Phi}}\right) d \mathbf{x} .
$$

Then the weak formulation for (3.1)-(3.2) is: Given $\mathbf{q} \in \mathbf{H}^{-1 / 2}\left(\operatorname{Div} ; \Gamma_{\rho}\right)$, find $\mathbf{v} \in$ $\mathbf{H}\left(\mathbf{c u r l} ; \Omega^{\mathrm{PML}}\right)$ such that $\hat{\mathbf{x}} \times \mathbf{v}=0$ on $\Gamma_{R}, \hat{\mathbf{x}} \times \mathbf{v}=\mathbf{q}$ on $\Gamma_{\rho}$, and

$$
c(\mathbf{v}, \boldsymbol{\Phi})=0, \quad \forall \boldsymbol{\Phi} \in \mathbf{H}_{0}\left(\operatorname{curl} ; \Omega^{\mathrm{PML}}\right) .
$$

We make the following assumption on the fictitious medium property $\sigma$, which is rather mild in the practical application of the PML technique

(H1) $\quad \sigma=\sigma_{0}\left(\frac{r-R}{\rho-R}\right)^{m}$ for some constant $\sigma_{0}>0$ and some integer $m \geq 1$.

From (H1) we know that $\beta(r)=1+1 \hat{\sigma}(r)$, where

$$
\hat{\sigma}(r)=\frac{1}{r} \int_{R}^{r} \sigma(t) d t=\frac{\sigma_{0}}{m+1} \frac{r-R}{r}\left(\frac{r-R}{\rho-R}\right)^{m} .
$$

Thus $\hat{\sigma} \leq \sigma$ for all $r \geq R$. Notice that $B A=\operatorname{diag}\left(\alpha \beta^{-2}, \alpha^{-1}, \alpha^{-1}\right)$; we have

$$
\begin{aligned}
& \operatorname{Re}[c(\boldsymbol{\Phi}, \boldsymbol{\Phi})] \\
= & \int_{\Omega^{\mathrm{PML}}}\left\{\frac{1-\hat{\sigma}^{2}+2 \sigma \hat{\sigma}}{\left(1+\hat{\sigma}^{2}\right)^{2}}\left|(\nabla \times \mathbf{\Phi})_{r}\right|^{2}+\frac{1}{|\alpha|^{2}}\left|(\nabla \times \mathbf{\Phi})_{\theta}\right|^{2}+\frac{1}{|\alpha|^{2}}\left|(\nabla \times \mathbf{\Phi})_{\phi}\right|^{2}\right\} d \mathbf{x} \\
& -k^{2} \int_{\Omega^{\mathrm{PML}}}\left\{\frac{1-\hat{\sigma}^{2}+2 \hat{\sigma} \sigma}{1+\sigma^{2}}\left|\mathbf{\Phi}_{r}\right|^{2}+\left|\boldsymbol{\Phi}_{\theta}\right|^{2}+\left|\boldsymbol{\Phi}_{\phi}\right|^{2}\right\} d \mathbf{x} .
\end{aligned}
$$

Since

$$
\frac{1-\hat{\sigma}^{2}+2 \sigma \hat{\sigma}}{\left(1+\hat{\sigma}^{2}\right)^{2}} \geq \frac{1+\sigma \hat{\sigma}}{\left(1+\hat{\sigma}^{2}\right)^{2}} \geq \frac{1}{1+\sigma_{0}^{2}}, \quad \frac{1-\hat{\sigma}^{2}+2 \hat{\sigma} \sigma}{1+\sigma^{2}} \geq \frac{1}{1+\sigma_{0}^{2}}
$$

we know by using the spectral theory of compact operators that (3.3) has a unique solution for every real $k$ except possibly for a discrete set of values of $k$ (see [12, Theorem 2] for a similar discussion on the PML equation for acoustic scattering problems). In this paper we will not elaborate on this issue and simply make the following assumption:

(H2) The Dirichlet PML problem in the layer (3.3) has a unique solution.

The uniqueness and existence of the Dirichlet PML problem for the acoustic scattering problem is proved in [8] for sufficiently large $\sigma_{0}>0$. How to extend the analysis in 8 to the electromagnetic PML problem in the layer is an interesting open problem. 
For any $\boldsymbol{\Phi} \in \mathbf{H}\left(\mathbf{c u r l} ; \Omega^{\mathrm{PML}}\right)$, we define

$$
\begin{aligned}
\|\boldsymbol{\Phi}\|_{*, \Omega^{\mathrm{PML}}}^{2}= & \int_{\Omega^{\mathrm{PML}}}\left[\frac{1+\sigma \hat{\sigma}}{(1+\hat{\sigma})^{2}}\left|(\nabla \times \boldsymbol{\Phi})_{r}\right|^{2}+\frac{1}{1+\sigma^{2}}\left|(\nabla \times \mathbf{\Phi})_{\theta}\right|^{2}\right. \\
& \left.+\frac{1}{1+\sigma^{2}}\left|(\nabla \times \mathbf{\Phi})_{\phi}\right|^{2}+k^{2}\left(\frac{1+\hat{\sigma} \sigma}{1+\sigma^{2}}\left|\mathbf{\Phi}_{r}\right|^{2}+\left|\mathbf{\Phi}_{\theta}\right|^{2}+\left|\mathbf{\Phi}_{\phi}\right|^{2}\right)\right] d \mathbf{x} .
\end{aligned}
$$

It is clear that $\|\boldsymbol{\Phi}\|_{*, \Omega^{\mathrm{PML}}}$ is an equivalent norm of $\mathbf{H}\left(\mathbf{c u r l} ; \Omega^{\mathrm{PML}}\right)$. Thus by (H2), there exists a constant $\hat{C}>0$ such that

$$
\sup _{\boldsymbol{\Psi} \in \mathbf{H}_{0}\left(\operatorname{curl} ; \Omega^{\mathrm{PML}}\right)} \frac{|c(\boldsymbol{\Phi}, \boldsymbol{\Phi})|}{\|\boldsymbol{\Phi}\|_{*, \Omega^{\mathrm{PML}}}} \geq \hat{C}\|\boldsymbol{\Psi}\|_{*, \Omega^{\mathrm{PML}}}, \quad \forall \boldsymbol{\Psi} \in \mathbf{H}_{0}\left(\mathbf{c u r l} ; \Omega^{\mathrm{PML}}\right) .
$$

Without loss of generality we assume $\hat{C} \leq 1$. The following theorem is the main result of this section.

Theorem 3.1. Let (H1)-(H2) be satisfied. Then for sufficiently large $\sigma_{0}>0$, the $P M L$ problem (2.10)-(2.11) has a unique solution $\hat{\mathbf{E}} \in \mathbf{H}\left(\mathbf{c u r l} ; \Omega_{\rho}\right)$. Moreover, we have the following estimate

$\|\mathbf{E}-\hat{\mathbf{E}}\|_{\mathbf{H}\left(\operatorname{curl} ; \Omega_{R}\right)} \leq C \hat{C}^{-1}(1+k R)^{3}\left|\alpha_{0}\right|^{3} e^{-\operatorname{Im}(k \tilde{\rho})\left(1-\frac{R^{2}}{|\hat{\rho}|^{2}}\right)^{1 / 2}}\|\hat{\mathbf{x}} \times \hat{\mathbf{E}}\|_{\mathbf{H}^{-1 / 2}\left(\operatorname{Div} ; \Gamma_{\mathrm{R}}\right)}$, where $\alpha_{0}=1+1 \sigma_{0}$.

The proof of this theorem will be given in section 3.3 which depends on the exponential decay estimates for the PML extension and the stability estimates of the Dirichlet problem of the PML equation in the layer.

3.1. Estimates for the PML extension. We first recall some estimates for the Hankel functions. The following estimate which is proved in 8$]$ based on the Macdonald formula will play an important role in our analysis.

Lemma 3.2. For any $\nu \in \mathbb{R}, z \in \mathbb{C}_{++}=\{z \in \mathbb{C}: \operatorname{Im}(z) \geq 0, \operatorname{Re}(z) \geq 0\}$ and $\Theta \in \mathbb{R}$ such that $0<\Theta<|z|$, we have

$$
\left|H_{\nu}^{(1)}(z)\right| \leq e^{-\operatorname{Im}(z)\left(1-\frac{\Theta^{2}}{|z|^{2}}\right)^{1 / 2}}\left|H_{\nu}^{(1)}(\Theta)\right| .
$$

We also need the following important estimate of the spherical Hankel functions [20, p.195].

Lemma 3.3. For any $\Theta>0, \delta_{n}(\Theta)=\frac{z_{n}^{(1)}(\Theta)}{h_{n}^{(1)}(\Theta)}$ satisfies $\left|\delta_{n}(\Theta)\right| \geq \frac{n(n+1)}{2 \Theta^{2}+n+1}$.

Lemma 3.4. For any $\lambda=\sum_{n=1}^{\infty} \sum_{m=-n}^{n} a_{n m} \mathbf{U}_{n}^{m}+b_{n m} \mathbf{V}_{n}^{m} \in \mathbf{H}^{-1 / 2}\left(\operatorname{Div} ; \Gamma_{\mathrm{R}}\right)$, let $\mathbb{E}(\lambda)(\tilde{r}, \hat{\mathbf{x}})$ be the PML extension in (2.9). Then we have

$$
\begin{aligned}
\frac{1}{1 k} \tilde{\nabla} \times \mathbb{E}(\lambda) & =\sum_{n=1}^{\infty} \sum_{m=-n}^{n} \frac{a_{m n} \tilde{\mathbf{N}}_{n}^{m}}{h_{n}^{(1)}(k R) \sqrt{n(n+1)}}-\frac{1 k R b_{m n} \tilde{\mathbf{M}}_{n}^{m}}{z_{n}^{(1)}(k R) \sqrt{n(n+1)}}, \\
\hat{\mathbf{x}} \times \mathbb{E}(\lambda) & =\sum_{n=1}^{\infty} \sum_{m=-n}^{n} \frac{h_{n}^{(1)}(k \tilde{r})}{h_{n}^{(1)}(k R)} a_{n m} \mathbf{U}_{n}^{m}+\frac{R}{\tilde{r}} \frac{z_{n}^{(1)}(k \tilde{r})}{z_{n}^{(1)}(k R)} b_{n m} \mathbf{V}_{n}^{m}, \\
\frac{1}{1 k} \hat{\mathbf{x}} \times(\tilde{\nabla} \times \mathbb{E}(\lambda)) & =\sum_{n=1}^{\infty} \sum_{m=-n}^{n} \frac{-1 k R b_{m n} h_{n}^{(1)}(k \tilde{r})}{z_{n}^{(1)}(k R)} \mathbf{U}_{n}^{m}+\frac{a_{m n} z_{n}^{(1)}(k \tilde{r})}{1 k \tilde{r} h_{n}^{(1)}(k R)} \mathbf{V}_{n}^{m}
\end{aligned}
$$


Proof. The first equality follows since $\frac{1}{1 k} \tilde{\nabla} \times \tilde{\mathbf{M}}_{n}^{m}=\tilde{\mathbf{N}}_{n}^{m}$ and $-\frac{1}{1 k} \tilde{\nabla} \times \tilde{\mathbf{N}}_{n}^{m}=\tilde{\mathbf{M}}_{n}^{m}$. From the definition of the vector spherical harmonics in (2.2), we derive from (2.7) and (2.8) that

$$
\begin{aligned}
\hat{\mathbf{x}} \times \tilde{\mathbf{M}}_{n}^{m} & =\sqrt{n(n+1)} h_{n}^{(1)}(k \tilde{r}) \mathbf{U}_{n}^{m}, \\
\hat{\mathbf{x}} \times \tilde{\mathbf{N}}_{n}^{m} & =\sqrt{n(n+1)} \frac{1}{1 k \tilde{r}} z_{n}^{(1)}(k \tilde{r}) \mathbf{V}_{n}^{m} .
\end{aligned}
$$

This proves the second and third equalities.

The following exponential decay estimate of the PML extension provides the first hint of the convergence of the PML problem (2.10)-(2.11).

Lemma 3.5. For any $\lambda \in \mathbf{H}^{-1 / 2}\left(\operatorname{Div} ; \Gamma_{\mathrm{R}}\right)$, let $\mathbb{E}(\lambda)$ be the PML extension in (2.9). Then, for any $r>R$, we have

$$
\|\hat{\mathbf{x}} \times \mathbb{E}(\lambda)\|_{\mathbf{H}^{-1 / 2}\left(\operatorname{Div} ; \Gamma_{\mathrm{r}}\right)} \leq C(1+k R) e^{-\operatorname{Im}(k \tilde{r})\left(1-\frac{R^{2}}{|\tilde{r}|^{2}}\right)^{1 / 2}}\|\lambda\|_{\mathbf{H}^{-1 / 2}\left(\operatorname{Div} ; \Gamma_{\mathbf{R}}\right)} .
$$

Proof. By Lemma 3.2 and using the relation $h_{n}^{(1)}(z)=\sqrt{\frac{\pi}{2 z}} H_{n+1 / 2}^{(1)}(z)$, we obtain

$$
\left|\frac{h_{n}^{(1)}(k \tilde{r})}{h_{n}^{(1)}(k R)}\right| \leq\left|\frac{R}{\tilde{r}}\right|^{1 / 2} e^{-\operatorname{Im}(k \tilde{r})\left(1-\frac{R^{2}}{|\tilde{r}|^{2}}\right)^{1 / 2}} \leq e^{-\operatorname{Im}(k \tilde{r})\left(1-\frac{R^{2}}{|\tilde{\mid}|^{2}}\right)^{1 / 2}} .
$$

Since $h_{n}^{(1)}(z)=-\frac{n+1}{z} h_{n}^{(1)}(z)+h_{n-1}^{(1)}(z)$, we have

$$
\begin{aligned}
\left|\frac{R}{\tilde{r}} \frac{z_{n}^{(1)}(k \tilde{r})}{z_{n}^{(1)}(k R)}\right| & =\left|\frac{R}{\tilde{r}} \frac{h_{n}^{(1)}(k \tilde{r})+k \tilde{r} h_{n}^{(1) \prime}(k \tilde{r})}{z_{n}^{(1)}(k R)}\right| \\
& =\left|\frac{R}{\tilde{r}} \frac{-n h_{n}^{(1)}(\tilde{r})+k \tilde{r} h_{n-1}^{(1)}(k \tilde{r})}{z_{n}^{(1)}(k R)}\right| \\
& \leq\left(n\left|\frac{h_{n}^{(1)}(k \tilde{r})}{h_{n}^{(1)}(k R)}\right|+k R\left|\frac{h_{n-1}^{(1)}(k \tilde{r})}{h_{n-1}^{(1)}(k R)} \frac{h_{n-1}^{(1)}(k R)}{h_{n}^{(1)}(k R)}\right|\right)\left|\frac{h_{n}^{(1)}(k R)}{z_{n}^{(1)}(k R)}\right| \\
& \leq(n+k R)\left|\delta_{n}(k R)\right|^{-1} e^{-\operatorname{Im}(k \tilde{r})\left(1-\frac{R^{2}}{|\tilde{r}|^{2}}\right)^{1 / 2}},
\end{aligned}
$$

where we use the inequality that $\left|h_{n-1}^{(1)}(k R)\right| \leq\left|h_{n}^{(1)}(k R)\right|$, which is a consequence of the Nicholson integral (see [8, (2.18)]).

It remains to estimate $\left|\delta_{n}(\Theta)\right|$ for $\Theta>0$. Since $h_{n}^{(1) \prime}(z)=\frac{n}{z} h_{n}^{(1)}(z)-h_{n+1}^{(1)}(z)$, we have

$$
\delta_{n}(\Theta)=\frac{z_{n}^{(1)}(\Theta)}{h_{n}^{(1)}(\Theta)}=n+1-\Theta \frac{h_{n+1}^{(1)}(\Theta)}{h_{n}^{(1)}(\Theta)},
$$

which implies, for $\Theta \geq 2 n+1,\left|\delta_{n}(\Theta)\right| \geq \Theta-(n+1) \geq n$ and thus $\left|\delta_{n}(\Theta)\right|^{-1} \leq n^{-1}$. For $\Theta \leq(2 n+1)$, we resort to the estimate of Nédeléc in Lemma 3.3 to get

$$
\left|\delta_{n}(\Theta)\right|^{-1} \leq \frac{1}{n}(1+4 \Theta), \quad \Theta \leq 2 n+1 .
$$

Hence

$$
(n+k R)\left|\delta_{n}(k R)\right|^{-1} \leq 3(1+4 k R) .
$$


Substitute it into (3.8); we conclude that

$$
\left|\frac{R}{\tilde{r}} \frac{z_{n}^{(1)}(k \tilde{r})}{z_{n}^{(1)}(k R)}\right| \leq C(1+k R) e^{-\operatorname{Im}(k \tilde{r})\left(1-\frac{R^{2}}{|\tilde{r}|^{2}}\right)^{1 / 2}} .
$$

The lemma now follows from the second equality in Lemma 3.4 by using the estimates (3.7), (3.10) and the definition (2.3).

3.2. The PML equation in the layer. In this subsection we derive the stability estimates for the Dirichlet problem of the PML equation in the layer. We need the following weighted norm of $\mathbf{H}\left(\operatorname{curl} ; \Omega^{\mathrm{PML}}\right)$ :

$$
\|\boldsymbol{\Phi}\|_{\text {curl } ; \Omega^{\mathrm{PML}}}=\left(\|\nabla \times \boldsymbol{\Phi}\|_{\mathbf{L}^{2}\left(\Omega^{\mathrm{PML}}\right)}^{2}+R^{-2}\|\boldsymbol{\Phi}\|_{\mathbf{L}^{2}\left(\Omega^{\mathrm{PML}}\right)}^{2}\right)^{1 / 2} .
$$

Lemma 3.6. Let (H1)-(H2) be satisfied. There exists a constant $C>0$ independent of $k, R, \rho$ and $\sigma_{0}$ such that the following estimates are satisfied:

$$
\begin{aligned}
& \|\mathbf{v}\|_{\mathbf{c u r l} ; \Omega^{\mathrm{PML}}} \leq C \hat{C}^{-1}\left|\alpha_{0}\right|^{2}\|\mathbf{q}\|_{\mathbf{H}^{-1 / 2}\left(\operatorname{Div} ; \Gamma_{\rho}\right)}, \\
& \|\hat{\mathbf{x}} \times(\nabla \times \mathbf{v})\|_{\mathbf{H}^{-1 / 2}\left(\operatorname{Div} ; \Gamma_{\mathrm{R}}\right)} \leq C \hat{C}^{-1}(1+k R)^{2}\left|\alpha_{0}\right|^{2}\|\mathbf{q}\|_{\mathbf{H}^{-1 / 2}\left(\operatorname{Div} ; \Gamma_{\rho}\right)},
\end{aligned}
$$

where $\alpha_{0}=1+1 \sigma_{0}$.

Proof. From the definition of the sesquilinear form $c$ it is easy to see that

$$
\begin{aligned}
|c(\mathbf{v}, \boldsymbol{\Phi})| \leq & \int_{\Omega^{\mathrm{PML}}}\left\{\left|\alpha \beta^{-2}\right|\left|(\nabla \times \mathbf{v})_{r} \|(\nabla \times \boldsymbol{\Phi})_{r}\right|\right. \\
& +|\alpha|^{-1}\left|(\nabla \times \mathbf{v})_{\theta}\right|\left|(\nabla \times \boldsymbol{\Phi})_{\theta}\right|+|\alpha|^{-1}\left|(\nabla \times \mathbf{v})_{\phi} \|(\nabla \times \boldsymbol{\Phi})_{\phi}\right| \\
& \left.+k^{2}\left(\left|\alpha^{-1} \beta^{2} \| \mathbf{v}_{r} \boldsymbol{\Phi}_{r}\right|+|\alpha|\left|\mathbf{v}_{\theta} \boldsymbol{\Phi}_{\theta}\right|+|\alpha|\left|\mathbf{v}_{\phi} \boldsymbol{\Phi}_{\phi}\right|\right)\right\} d \mathbf{x} \\
\leq & C(1+k R)\left|\alpha_{0}\right|\|\mathbf{v}\|_{*, \Omega^{\mathrm{PML}}}\|\boldsymbol{\Phi}\|_{\text {curl } ; \Omega^{\mathrm{PML}}} .
\end{aligned}
$$

To show (3.11), let $\boldsymbol{\Psi} \in \mathbf{H}\left(\mathbf{c u r l} ; \Omega^{\mathrm{PML}}\right)$ be such that $\hat{\mathbf{x}} \times \mathbf{\Psi}=0$ on $\Gamma_{R}$ and $\hat{\mathbf{x}} \times \mathbf{\Psi}=\mathbf{q}$ on $\Gamma_{\rho}$; then $\boldsymbol{\Phi}=\mathbf{v}-\boldsymbol{\Psi} \in \mathbf{H}_{0}\left(\operatorname{curl} ; \Omega^{\mathrm{PML}}\right)$. Thus $c(\mathbf{v}, \mathbf{v}-\mathbf{\Psi})=0$ by (3.3), and consequently

$$
|c(\mathbf{v}, \mathbf{v})|=|c(\mathbf{v}, \Psi)| \leq C(1+k R)\left|\alpha_{0}\right|\|\mathbf{v}\|_{*, \Omega^{\mathrm{PML}}}\|\Psi\|_{\mathbf{c u r l} ; \Omega^{\mathrm{PML}}} .
$$

Thus by (3.4),

$$
\|\mathbf{v}\|_{*, \Omega^{\mathrm{PML}}} \leq C \hat{C}^{-1}(1+k R)\left|\alpha_{0}\right|\|\boldsymbol{\Psi}\|_{\mathbf{c u r l} ; \Omega^{\mathrm{PML}}},
$$

for any $\boldsymbol{\Psi}$ such that $\hat{\mathbf{x}} \times \boldsymbol{\Psi}=0$ on $\Gamma_{R}$ and $\hat{\mathbf{x}} \times \boldsymbol{\Psi}=\mathbf{q}$ on $\Gamma_{\rho}$. By the trace theorem, we obtain

$$
\|\mathbf{v}\|_{*, \Omega^{\mathrm{PML}}} \leq C \hat{C}^{-1}(1+k R)\left|\alpha_{0}\right|\|\mathbf{q}\|_{\mathbf{H}^{-1 / 2}\left(\operatorname{Div} ; \Gamma_{\rho}\right)} .
$$

This proves (3.11) since

$$
\begin{aligned}
\|\mathbf{v}\|_{*, \Omega^{\mathrm{PML}}}^{2} & \geq\left|\alpha_{0}\right|^{-2}\|\nabla \times \mathbf{v}\|_{L^{2}\left(\Omega^{\mathrm{PML}}\right)}+k^{2}\left|\alpha_{0}\right|^{-2}\|\mathbf{v}\|_{L^{2}\left(\Omega^{\mathrm{PML}}\right)} \\
& \geq\left|\alpha_{0}\right|^{-2}\left(1+k^{2} R^{2}\right)\|\mathbf{v}\|_{\mathbf{c u r l} ; \Omega^{\mathrm{PML}}}^{2} .
\end{aligned}
$$

To show (3.12), noticing that $\mathbf{v}$ satisfies the differential equation (3.1), we integrate by parts to get, for any $\boldsymbol{\Phi} \in \mathbf{H}\left(\operatorname{curl} ; \Omega^{\mathrm{PML}}\right)$ such that $\hat{\mathbf{x}} \times \mathbf{\Phi}=0$ on $\Gamma_{\rho}$,

$$
c(\mathbf{v}, \mathbf{\Phi})=-\langle\hat{\mathbf{x}} \times(\nabla \times \mathbf{v}),(\hat{\mathbf{x}} \times \mathbf{\Phi}) \times \hat{\mathbf{x}}\rangle_{\Gamma_{R}} .
$$


Thus, by (3.13) and (3.14),

$$
\begin{aligned}
\|\hat{\mathbf{x}} \times(\nabla \times \mathbf{v})\|_{\mathbf{H}^{-1 / 2}\left(\operatorname{Div} ; \Gamma_{\mathrm{R}}\right)} & \leq \sup _{\mathbf{\Phi} \in \mathbf{H}_{D}\left(\operatorname{curl} ; \Omega^{\mathrm{PML}}\right)} \frac{|c(\mathbf{v}, \boldsymbol{\Phi})|}{\|\mathbf{\Phi}\|_{\mathbf{c u r l} ; \Omega^{\mathrm{PML}}}} \\
& \leq C(1+k R)\left|\alpha_{0}\right|\|\mathbf{v}\|_{*, \Omega^{\mathrm{PML}}} \\
& \leq C \hat{C}^{-1}(1+k R)^{2}\left|\alpha_{0}\right|^{2}\|\mathbf{q}\|_{\mathbf{H}^{-1 / 2}\left(\operatorname{Div} ; \Gamma_{\rho}\right)} .
\end{aligned}
$$

This concludes the proof.

3.3. Convergence of the PML problem. We start by introducing the propagation operator $\mathbb{P}: \mathbf{H}^{-1 / 2}\left(\operatorname{Div} ; \Gamma_{\mathrm{R}}\right) \rightarrow \mathbf{H}^{-1 / 2}\left(\operatorname{Div} ; \Gamma_{\rho}\right)$. For any $\lambda \in \mathbf{H}^{-1 / 2}\left(\operatorname{Div} ; \Gamma_{\mathrm{R}}\right)$, we define $\mathbb{P}(\lambda)=\hat{\mathbf{x}} \times \mathbb{E}(\lambda)(\tilde{\rho}, \hat{\mathbf{x}})$, where $\mathbb{E}(\lambda)$ is the PML extension of $\lambda$ in (2.9). By Lemma 3.5, we know that

$$
\|\mathbb{P}(\lambda)\|_{\mathbf{H}^{-1 / 2}\left(\operatorname{Div} ; \Gamma_{\rho}\right)} \leq C(1+k R) e^{-\operatorname{Im}(k \tilde{\rho})\left(1-\frac{R^{2}}{|\tilde{\rho}|^{2}}\right)^{1 / 2}}\|\lambda\|_{\mathbf{H}^{-1 / 2}\left(\operatorname{Div} ; \Gamma_{\mathrm{R}}\right)} .
$$

Lemma 3.7. Let $(\mathbf{H} 1)-(\mathbf{H} 2)$ be satisfied. We have

$$
\begin{aligned}
& \left\|1 k\left(\hat{G}_{e}-G_{e}\right)(\lambda)\right\|_{\mathbf{H}^{-1 / 2}\left(\operatorname{Div} ; \Gamma_{\mathrm{R}}\right)} \\
\leq & C \hat{C}^{-1}(1+k R)^{3}\left|\alpha_{0}\right|^{3} e^{-\operatorname{Im}(k \tilde{\rho})\left(1-\frac{R^{2}}{|\tilde{\rho}|^{2}}\right)^{1 / 2}}\|\lambda\|_{\mathbf{H}^{-1 / 2}\left(\operatorname{Div} ; \Gamma_{\mathrm{R}}\right)} .
\end{aligned}
$$

Proof. For any $\lambda \in \mathbf{H}^{-1 / 2}$ (Div; $\Gamma_{\mathrm{R}}$ ), by (2.12) and the third equality in Lemma 3.4. we know that

$$
G_{e}(\lambda)=\frac{1}{1 k} \hat{\mathbf{x}} \times\left.(\tilde{\nabla} \times \mathbb{E}(\lambda))\right|_{\Gamma_{R}} .
$$

Since $\tilde{\nabla} \times \mathbb{E}(\lambda)=A \nabla \times B \mathbb{E}(\lambda)$ and $A=\operatorname{diag}\{1,1,1\}$ on $\Gamma_{R}$, we have

$$
G_{e}(\lambda)=\frac{1}{1 k} \hat{\mathbf{x}} \times\left.(\nabla \times B \mathbb{E}(\lambda))\right|_{\Gamma_{R}} .
$$

Now by (2.16) - (2.17), we know that $\left(G_{e}-\hat{G}_{e}\right)(\lambda)=\frac{1}{1 k} \hat{\mathbf{x}} \times(\nabla \times \mathbf{w})$, where w satisfies

$$
\begin{aligned}
& \nabla \times B A(\nabla \times \mathbf{w})-k^{2}(B A)^{-1} \mathbf{w}=0 \text { in } \Omega^{\mathrm{PML}}, \\
& \hat{\mathbf{x}} \times \mathbf{w}=0 \text { on } \Gamma_{R}, \quad \hat{\mathbf{x}} \times \mathbf{w}=B \mathbb{P}(\lambda) \text { on } \Gamma_{\rho} .
\end{aligned}
$$

By Lemma 3.6 and (3.15), we have

$$
\begin{aligned}
& \|\hat{\mathbf{x}} \times(\nabla \times B \mathbf{w})\|_{\mathbf{H}^{-1 / 2}\left(\operatorname{Div} ; \Gamma_{\mathrm{R}}\right)} \\
\leq & C \hat{C}^{-1}(1+k R)^{2}\left|\alpha_{0}\right|^{2}\|B \mathbb{P}(\lambda)\|_{\mathbf{H}^{-1 / 2}\left(\operatorname{Div} ; \Gamma_{\rho}\right)} \\
\leq & C \hat{C}^{-1}(1+k R)^{3}\left|\alpha_{0}\right|^{3} e^{-\operatorname{Im}(k \tilde{\rho})\left(1-\frac{R^{2}}{|\tilde{\rho}|^{2}}\right)^{1 / 2}}\|\lambda\|_{\mathbf{H}^{-1 / 2}\left(\operatorname{Div} ; \Gamma_{\mathrm{R}}\right)} .
\end{aligned}
$$

This completes the proof.

Now we are ready to prove the main theorem of this section.

Proof of Theorem 3.1. The existence of a unique solution for (2.18) follows from Lemma 3.7 by using the small perturbation argument the same as that in [10, Theorem 2.4]. Next, by (2.13) and (2.18) we have, for any $\boldsymbol{\Phi} \in \mathbf{H}_{D}\left(\mathbf{c u r l} ; \Omega_{R}\right)$,

$$
a(\mathbf{E}-\hat{\mathbf{E}}, \boldsymbol{\Phi})=\hat{a}(\hat{\mathbf{E}}, \boldsymbol{\Phi})-a(\hat{\mathbf{E}}, \boldsymbol{\Phi})=1 k\left\langle\left(\hat{G}_{e}-G_{e}\right)(\hat{\mathbf{x}} \times \hat{\mathbf{E}}),(\hat{\mathbf{x}} \times \boldsymbol{\Phi}) \times \hat{\mathbf{x}}\right\rangle_{\Gamma_{R}} .
$$

This yields the desired estimate upon using Lemma 3.7 and (2.14). 


\section{Finite Element Discretization}

We start by introducing the weak formulation of the PML problem (2.10) $-(2.11)$. Let

$$
b(\boldsymbol{\Psi}, \boldsymbol{\Phi})=\int_{\Omega_{\rho}}\left(B A \nabla \times \mathbf{\Psi} \cdot \nabla \times \overline{\mathbf{\Phi}}-k^{2}(B A)^{-1} \mathbf{\Psi} \cdot \overline{\mathbf{\Phi}}\right) d \mathbf{x} .
$$

Then the weak formulation of (2.10)-(2.11) is: Given $\mathbf{g} \in \mathbf{H}^{-1 / 2}\left(\mathrm{Div} ; \Gamma_{\mathrm{D}}\right)$, find $\hat{\mathbf{E}} \in \mathbf{H}\left(\mathbf{c u r l}, \Omega_{\rho}\right)$, such that $\mathbf{n} \times \hat{\mathbf{E}}=\mathbf{g}$ on $\Gamma_{D}, \hat{\mathbf{x}} \times \hat{\mathbf{E}}=0$ on $\Gamma_{\rho}$, and

$$
b(\hat{\mathbf{E}}, \boldsymbol{\Phi})=0, \quad \forall \boldsymbol{\Phi} \in \mathbf{H}_{0}\left(\operatorname{curl} ; \Omega_{\rho}\right) .
$$

Let $\Gamma_{\rho}^{h}$, which consists of piecewise triangles whose vertices lie on $\Gamma_{\rho}$, be an approximation of $\Gamma_{\rho}$. Let $\Omega_{\rho}^{h}$ be the subdomain of $\Omega_{\rho}$ bounded by $\Gamma_{D}$ and $\Gamma_{\rho}^{h}$. Let $\mathcal{M}_{h}$ be a regular triangulation of the domain $\Omega_{\rho}^{h}$. We will use the lowest order Nédeléc edge element [19] for which the finite element space $\mathbf{U}_{h}$ over $\mathcal{M}_{h}$ is defined by

$$
\mathbf{U}_{h}=\left\{\mathbf{u} \in \mathbf{H}\left(\operatorname{curl} ; \Omega_{\rho}^{h}\right):\left.\mathbf{u}\right|_{K}=\mathbf{a}_{K}+\mathbf{b}_{K} \times \mathbf{x}, \forall \mathbf{a}_{K}, \mathbf{b}_{K} \in \mathbb{R}^{3}, \forall K \in \mathcal{M}_{h}\right\}
$$

Degrees of freedom of functions $\mathbf{u} \in \mathbf{U}_{h}$ on every $K \in \mathcal{M}_{h}$ are $\int_{e_{i}} \mathbf{u} \cdot \mathbf{d l}, i=1, \ldots, 6$, where $e_{1}, \ldots, e_{6}$ are the six edges of $K$. Denote $\stackrel{\circ}{\mathbf{U}}_{h}=\mathbf{U}_{h} \cap \mathbf{H}_{0}\left(\mathbf{c u r l} ; \Omega_{\rho}^{h}\right)$. In the following, we will always assume that the functions in $\stackrel{\circ}{\mathbf{U}}_{h}$ are extended to the domain $\Omega_{\rho}$ by zero so that any function $\mathbf{u} \in \stackrel{\circ}{\mathbf{U}}_{h}$ is also a function in $\mathbf{H}_{0}\left(\mathbf{c u r l} ; \Omega_{\rho}\right)$. The finite element approximation to (4.2) reads as follows: Find $\mathbf{E}_{h} \subset \mathbf{U}_{h}$ such that $\mathbf{n} \times \mathbf{E}_{h}=\mathbf{g}_{h}$ on $\Gamma_{D}, \mathbf{n} \times \mathbf{E}_{h}=0$ on $\Gamma_{\rho}^{h}$, and

$$
b\left(\mathbf{E}_{h}, \boldsymbol{\Phi}_{h}\right)=0, \quad \forall \boldsymbol{\Phi}_{h} \in \stackrel{\circ}{\mathbf{U}}_{h}
$$

Here $\mathbf{g}_{h}$ is some edge element approximation of $\mathbf{g}$ on $\Gamma_{D}$. Notice that the integral in $b\left(\mathbf{E}_{h}, \boldsymbol{\Phi}_{h}\right)$ is actually over $\Omega_{\rho}^{h}$ since $\boldsymbol{\Phi}_{h}=0$ in $\Omega_{\rho} \backslash \Omega_{\rho}^{h}$ by our convention. The existence and uniqueness of the discrete problem (4.3) is a difficult problem due to the non-coerciveness of the sesquilinear form $b: \mathbf{H}\left(\operatorname{curl} ; \Omega_{\rho}\right) \times \mathbf{H}\left(\operatorname{curl} ; \Omega_{\rho}\right) \rightarrow$ $\mathbb{C}$. Based on a general argument in Schatz [21], the unique existence of (4.3) for sufficiently small mesh size $h<h^{*}$ can be proved by using the unique existence of the continuous problem (4.2). In this paper we are interested in a posteriori error estimates and the associated adaptive algorithm. Thus in the following, we simply assume the discrete problem (4.3) has a unique solution $\mathbf{E}_{h}$.

For any $K \in \mathcal{M}_{h}$, we denote by $h_{K}$ its diameter. Let $\mathcal{F}_{h}$ be the set of all faces of the mesh $\mathcal{M}_{h}$ that do not lie on $\Gamma_{D}$ and $\Gamma_{\rho}^{h}$. For any $F \in \mathcal{F}_{h}, h_{F}$ stands for its diameter. For any interior face $F$ which is a common face of $K_{1}$ and $K_{2}$ in $\mathcal{M}_{h}$, we define the following jump residuals across $F$ :

$$
\begin{aligned}
& \llbracket \mathbf{n} \times\left(B A \nabla \times \mathbf{E}_{h}\right) \rrbracket=\mathbf{n}_{F} \times\left(B A \nabla \times\left(\left.\mathbf{E}_{h}\right|_{K_{1}}-\left.\mathbf{E}_{h}\right|_{K_{2}}\right)\right), \\
& \llbracket k^{2}(B A)^{-1} \mathbf{E}_{h} \cdot \mathbf{n} \rrbracket=k^{2}(B A)^{-1}\left(\left.\mathbf{E}_{h}\right|_{K_{1}}-\left.\mathbf{E}_{h}\right|_{K_{2}}\right) \cdot \mathbf{n}_{F},
\end{aligned}
$$


using the convention that the unit norm vector $\mathbf{n}_{F}$ to $F$ points from $K_{2}$ to $K_{1}$. The local error indicator $\eta_{K}$ for any $K \in \mathcal{M}_{h}$ is defined as

$$
\begin{aligned}
\eta_{K}^{2}= & h_{K}^{2}\left\|k^{2}(B A)^{-1} \mathbf{E}_{h}-\nabla \times\left(B A \nabla \times \mathbf{E}_{h}\right)\right\|_{\mathbf{L}^{2}(K)}^{2} \\
+ & h_{K}^{2}\left\|\operatorname{div}\left(k^{2}(B A)^{-1} \mathbf{E}_{h}\right)\right\|_{L^{2}(K)}^{2} \\
+ & h_{K}\left\|\llbracket \mathbf{n} \times\left(B A \nabla \times \mathbf{E}_{h}\right) \rrbracket\right\|_{\mathbf{L}^{2}(\partial K)}^{2}+h_{K}\left\|\llbracket k^{2}(B A)^{-1} \mathbf{E}_{h} \cdot \mathbf{n} \rrbracket\right\|_{L^{2}(\partial K)}^{2} .
\end{aligned}
$$

The following theorem is the main result of this paper.

Theorem 4.1. Let (H1)-(H2) be satisfied. There exists a constant $C$ depending only on the minimum angle of the mesh $\mathcal{M}_{h}$ such that the following a posteriori error estimate is valid:

$$
\begin{aligned}
& \left\|\mathbf{E}-\mathbf{E}_{h}\right\|_{\mathbf{H}\left(\mathbf{c u r l} ; \Omega_{R}\right)} \\
\leq & C\left\|\mathbf{g}-\mathbf{g}_{h}\right\|_{\mathbf{H}^{-1 / 2}\left(\operatorname{Div} ; \Gamma_{\mathrm{D}}\right)}+C \hat{C}^{-1}\left|\alpha_{0}\right|^{3}(1+k R)^{3} R^{1 / 2}\left(\sum_{K \in \mathcal{M}_{h}} \eta_{K}^{2}\right)^{1 / 2} \\
& +C \hat{C}^{-1}\left|\alpha_{0}\right|^{3}(1+k R)^{3} e^{-\operatorname{Im}(k \tilde{\rho})\left(1-\frac{R^{2}}{|\tilde{\rho}|^{2}}\right)^{1 / 2}}\left\|\hat{\mathbf{x}} \times \mathbf{E}_{h}\right\|_{\mathbf{H}^{-1 / 2}\left(\operatorname{Div} ; \Gamma_{\mathrm{R}}\right)} .
\end{aligned}
$$

The proof of this theorem will be given in Section 5.3. One of the key ingredients of the a posteriori error analysis is the Birman-Solomyak decomposition theorem in Lipschitz domains 6, 14, 9]. More precisely, the following result whose proof can be found in [14, 9 will be used.

Lemma 4.2. For any $\mathbf{u} \in \mathbf{H}_{0}\left(\mathbf{c u r l}, \Omega_{\rho}\right)$, there exists a $\mathbf{\Phi} \in \mathbf{H}_{0}\left(\operatorname{curl}, \Omega_{\rho}\right) \cap H^{1}\left(\Omega_{\rho}\right)^{3}$ and $a \varphi \in H_{0}^{1}\left(\Omega_{\rho}\right)$ such that $\mathbf{u}=\mathbf{\Phi}+\nabla \varphi$ in $\Omega_{\rho}$, and

$$
\|\varphi\|_{H^{1}\left(\Omega_{\rho}\right)}+\|\mathbf{\Phi}\|_{\mathbf{H}^{1}\left(\Omega_{\rho}\right)} \leq C\|\mathbf{u}\|_{\mathbf{H}\left(\mathbf{c u r l} ; \Omega_{\rho}\right)} .
$$

Let $V_{h}$ be the standard $H^{1}$-conforming piecewise linear finite element space over $\mathcal{M}_{h}$ and $\stackrel{\circ}{V}_{h}=H_{0}^{1}\left(\Omega_{\rho}^{h}\right) \cap V_{h}$. In the following, we will also assume that the functions in $\stackrel{\circ}{V}_{h}$ are extended to the domain $\Omega_{\rho}$ by zero so that any function in $\stackrel{\circ}{V}_{h}$ is also a function in $H_{0}^{1}\left(\Omega_{\rho}\right)$. In $\S 5.3$, we will use the Clément operator $r_{h}: H_{0}^{1}\left(\Omega_{\rho}\right) \rightarrow \stackrel{\circ}{V}_{h}[11$ and the Beck-Hiptmair-Hoppe-Wohlmuth interpolation operator $\pi_{h}: H^{1}\left(\Omega_{\rho}\right)^{3} \cap \mathbf{H}_{0}\left(\mathbf{c u r l} ; \Omega_{\rho}\right) \rightarrow \stackrel{\circ}{\mathbf{U}}_{h}[\underline{4}$ which satisfy the following estimates:

$$
\left\|\varphi-r_{h} \varphi\right\|_{L^{2}(K)} \leq C h_{K}\|\nabla \varphi\|_{\mathbf{L}^{2}(\tilde{K})},\left\|\varphi-r_{h} \varphi\right\|_{L^{2}(F)} \leq C h_{F}^{1 / 2}\|\nabla \varphi\|_{\mathbf{L}^{2}(\tilde{F})},
$$

$$
\left\|\boldsymbol{\Phi}-\pi_{h} \boldsymbol{\Phi}\right\|_{\mathbf{L}^{2}(K)} \leq C h_{K}\|\nabla \boldsymbol{\Phi}\|_{\mathbf{L}^{2}(\tilde{K})},\left\|\boldsymbol{\Phi}-\pi_{h} \boldsymbol{\Phi}\right\|_{\mathbf{L}^{2}(F)} \leq C h_{F}^{1 / 2}\|\nabla \boldsymbol{\Phi}\|_{\mathbf{L}^{2}(\tilde{F})},
$$

where $\tilde{A}$ is the union of elements on $\mathcal{M}_{h}$ with non-empty intersection with $A$, $A=K \in \mathcal{M}_{h}$ or $F \in \mathcal{F}_{h}$.

\section{A POSTERIORI ERROR ANALYSIS}

In this section, we prove the a posteriori error estimates in Theorem 4.1. To begin with, let $\boldsymbol{\Psi} \in \mathbf{H}\left(\mathbf{c u r l} ; \Omega_{R}\right)$ such that $\mathbf{n} \times \mathbf{\Psi}=\mathbf{g}-\mathbf{g}_{h}$ on $\Gamma_{D}$; then $\mathbf{E}-\mathbf{E}_{h}-\boldsymbol{\Psi} \in$ $\mathbf{H}_{D}\left(\operatorname{curl} ; \Omega_{R}\right)$. Thus by (2.14) we have

$$
\left\|\mathbf{E}-\mathbf{E}_{h}-\boldsymbol{\Psi}\right\|_{\mathbf{H}\left(\mathbf{c u r l} ; \Omega_{R}\right)} \leq C \sup _{\mathbf{\Phi} \in \mathbf{H}_{D}\left(\operatorname{curl} ; \Omega_{R}\right)} \frac{\left|a\left(\mathbf{E}-\mathbf{E}_{h}-\mathbf{\Psi}, \mathbf{\Phi}\right)\right|}{\|\boldsymbol{\Phi}\|_{\mathbf{H}\left(\mathbf{c u r l} ; \Omega_{R}\right)}} .
$$


Since $|a(\boldsymbol{\Psi}, \boldsymbol{\Phi})| \leq C\|\boldsymbol{\Psi}\|_{\mathbf{H}\left(\operatorname{curl} ; \Omega_{R}\right)}\|\boldsymbol{\Phi}\|_{\mathbf{H}\left(\operatorname{curl} ; \Omega_{R}\right)}$, we obtain

$$
\left\|\mathbf{E}-\mathbf{E}_{h}\right\|_{\mathbf{H}\left(\operatorname{curl} ; \Omega_{R}\right)} \leq C\|\Psi\|_{\mathbf{H}\left(\operatorname{curl} ; \Omega_{R}\right)}+C \sup _{\mathbf{\Phi} \in \mathbf{H}_{D}\left(\operatorname{curl} ; \Omega_{R}\right)} \frac{\left|a\left(\mathbf{E}-\mathbf{E}_{h}, \boldsymbol{\Phi}\right)\right|}{\|\mathbf{\Phi}\|_{\mathbf{H}\left(\operatorname{curl} ; \Omega_{R}\right)}} .
$$

The above estimate is valid for any $\boldsymbol{\Psi} \in \mathbf{H}\left(\mathbf{c u r l} ; \Omega_{R}\right)$ such that $\mathbf{n} \times \mathbf{\Psi}=\mathbf{g}-\mathbf{g}_{h}$ on $\Gamma_{D}$, we get by the trace theorem

$$
\begin{aligned}
\left\|\mathbf{E}-\mathbf{E}_{h}\right\|_{\mathbf{H}\left(\mathbf{c u r l} ; \Omega_{R}\right)} \leq & C\left\|\mathbf{g}-\mathbf{g}_{h}\right\|_{\mathbf{H}^{-1 / 2}\left(\operatorname{Div} ; \Gamma_{\mathrm{D}}\right)} \\
+ & C \sup _{\mathbf{\Phi} \in \mathbf{H}_{D}\left(\mathbf{c u r l} ; \Omega_{R}\right)} \frac{\left|a\left(\mathbf{E}-\mathbf{E}_{h}, \boldsymbol{\Phi}\right)\right|}{\|\mathbf{\Phi}\|_{\mathbf{H}\left(\operatorname{curl} ; \Omega_{R}\right)}} .
\end{aligned}
$$

5.1. Error representation formula. For any $\boldsymbol{\Phi} \in \mathbf{H}_{D}\left(\operatorname{curl} ; \Omega_{R}\right)$ which satisfies $\mathbf{n} \times \mathbf{\Phi}=0$ on $\Gamma_{D}$, we extend $\boldsymbol{\Phi}$ to $\Omega^{\mathrm{PML}}$, denoted by $\hat{\boldsymbol{\Phi}}$, through the following conditions:

$$
\begin{aligned}
& \nabla \times \overline{B A} \nabla \times \hat{\boldsymbol{\Phi}}-k^{2}(\overline{B A})^{-1} \hat{\boldsymbol{\Phi}}=0 \quad \text { in } \Omega^{\mathrm{PML}}, \\
& \hat{\mathbf{x}} \times \hat{\boldsymbol{\Phi}}=\hat{\mathbf{x}} \times \mathbf{\Phi} \quad \text { on } \Gamma_{R}, \quad \hat{\mathbf{x}} \times \hat{\mathbf{\Phi}}=0 \quad \text { on } \Gamma_{\rho} .
\end{aligned}
$$

By (H2) we know that $\hat{\boldsymbol{\Phi}}$ is well-defined.

Lemma 5.1. Let $(\mathbf{H 2})$ be satisfied. For any $\mathbf{\Phi}, \mathbf{\Psi} \in \mathbf{H}\left(\mathbf{c u r l} ; \Omega_{R}\right)$, we have

$$
\left\langle\hat{G}_{e}(\hat{\mathbf{x}} \times \mathbf{\Phi}),(\hat{\mathbf{x}} \times \mathbf{\Psi}) \times \hat{\mathbf{x}}\right\rangle_{\Gamma_{R}}=\left\langle\hat{G}_{e}(\hat{\mathbf{x}} \times \overline{\mathbf{\Psi}}),(\hat{\mathbf{x}} \times \overline{\mathbf{\Phi}}) \times \hat{\mathbf{x}}\right\rangle_{\Gamma_{R}} .
$$

Proof. By the definition of the approximate Calderon operator $\hat{G}_{e}$ in (2.16)-(2.17) we know that

$$
\hat{G}_{e}\left(\hat{\mathbf{x}} \times\left.\mathbf{\Phi}\right|_{\Gamma_{R}}\right)=\frac{1}{1 k} \hat{\mathbf{x}} \times\left.(\nabla \times \mathbf{w})\right|_{\Gamma_{R}},
$$

where $\mathbf{w}$ satisfies

$$
\begin{aligned}
& \nabla \times B A \nabla \times \mathbf{w}-k^{2}(B A)^{-1} \mathbf{w}=0 \quad \text { in } \Omega^{\mathrm{PML}}, \\
& \hat{\mathbf{x}} \times \mathbf{w}=\hat{\mathbf{x}} \times \mathbf{\Phi} \text { on } \Gamma_{R}, \quad \hat{\mathbf{x}} \times \mathbf{w}=0 \text { on } \Gamma_{\rho} .
\end{aligned}
$$

The solution of (5.4)-(5.5) can be represented as

$$
\mathbf{w}=\sum_{n=1}^{\infty} \sum_{m=-n}^{n} \alpha_{n m} \mathbf{M}_{n}^{m}(\tilde{r}, \hat{\mathbf{x}})+\beta_{n m} \mathbf{N}_{n}^{m}(\tilde{r}, \hat{\mathbf{x}})+\alpha_{n m}^{\prime} \hat{\mathbf{M}}_{n}^{m}(\tilde{r}, \hat{\mathbf{x}})+\beta_{n m}^{\prime} \hat{\mathbf{N}}_{n}^{m}(\tilde{r}, \hat{\mathbf{x}}),
$$

where, corresponding to $\mathbf{M}_{n}^{m}, \mathbf{N}_{n}^{m}$ for $h_{n}^{(1)}(k \tilde{r})$ in (2.7)-(2.8),

$$
\begin{aligned}
\hat{\mathbf{M}}_{n}^{m}(\tilde{r}, \hat{\mathbf{x}}) & =h_{n}^{(2)}(k \tilde{r}) \nabla_{\partial B_{1}} Y_{n}^{m}(\hat{\mathbf{x}}) \times \hat{\mathbf{x}} \\
\hat{\mathbf{N}}_{n}^{m}(\tilde{r}, \hat{\mathbf{x}}) & =\frac{1}{1 k} \tilde{\nabla} \times \hat{\mathbf{M}}_{n}^{m}(\tilde{r}, \hat{\mathbf{x}}) \\
& =\frac{\sqrt{n(n+1)}}{1 k \tilde{r}} z_{n}^{(2)}(k \tilde{r}) \mathbf{U}_{n}^{m}(\hat{\mathbf{x}})+\frac{n(n+1)}{1 k \tilde{r}} h_{n}^{(2)}(k \tilde{r}) Y_{n}^{m}(\hat{\mathbf{x}}) \hat{\mathbf{x}} .
\end{aligned}
$$

Here $z_{n}^{(2)}(k \tilde{r})=h_{n}^{(2)}(k \tilde{r})+k \tilde{r} h_{n}^{(2) \prime}(k \tilde{r})$ and $h_{n}^{(2)}(z)$ is the spherical Hankel function of the second kind of order $n$. The constants $\alpha_{n m}, \beta_{n m}, \alpha_{n m}^{\prime}, \beta_{n m}^{\prime}$ are determined by 
the boundary conditions (5.3). By (3.5) $-\left(\underline{3.6)}\right.$ and similar identities for $\hat{\mathbf{M}}_{n}^{m}, \hat{\mathbf{N}}_{n}^{m}$, we know that

$$
\begin{array}{r}
\hat{\mathbf{x}} \times \mathbf{w}=\sum_{n=1}^{\infty} \sum_{m=-n}^{n} \sqrt{n(n+1)}\left(\alpha_{n m} h_{n}^{(1)}(k \tilde{r})+\alpha_{n m}^{\prime} h_{n}^{(2)}(k \tilde{r})\right) \mathbf{U}_{n}^{m}(\hat{\mathbf{x}}) \\
+\sqrt{n(n+1)} \frac{1}{\tilde{r}}\left(\beta_{n m} z_{n}^{(1)}(k \tilde{r})+\beta_{n m}^{\prime} z_{n}^{(2)}(k \tilde{r})\right) \mathbf{V}_{n}^{m}(\hat{\mathbf{x}}) .
\end{array}
$$

If $\hat{\mathbf{x}} \times\left.\boldsymbol{\Phi}\right|_{\Gamma_{R}}=\sum_{n=1}^{\infty} \sum_{m=-n}^{n} a_{n m} \mathbf{U}_{n}^{m}+b_{n m} \mathbf{V}_{n}^{m}$, then (5.3) implies

$$
\begin{aligned}
& \alpha_{n m} h_{n}^{(1)}(k R)+\alpha_{n m}^{\prime} h_{n}^{(2)}(k R)=\frac{a_{n m}}{\sqrt{n(n+1)}}, \quad \alpha_{n m} h_{n}^{(1)}(k \tilde{\rho})+\alpha_{n m}^{\prime} h_{n}^{(2)}(k \tilde{\rho})=0, \\
& \beta_{n m} z_{n}^{(1)}(k R)+\beta_{n m}^{\prime} z_{n}^{(2)}(k R)=\frac{R b_{n m}}{\sqrt{n(n+1)}}, \quad \beta_{n m} z_{n}^{(1)}(k \tilde{\rho})+\beta_{n m}^{\prime} z_{n}^{(2)}(k \tilde{\rho})=0 .
\end{aligned}
$$

By (H2), the problem (5.2)-(5.3) has a unique solution, and consequently the above linear system of equations for $\alpha_{m n}, \alpha_{m n}^{\prime}, \beta_{m n}, \beta_{m n}^{\prime}$ has a unique solution. Denote by

$$
\begin{aligned}
& H_{n}=h_{n}^{(1)}(k R) h_{n}^{(2)}(k \tilde{\rho})-h_{n}^{(2)}(k R) h_{n}^{(1)}(k \tilde{\rho}), \\
& I_{n}=z_{n}^{(1)}(k R) z_{n}^{(2)}(k \tilde{\rho})-z_{n}^{(2)}(k R) z_{n}^{(1)}(k \tilde{\rho})
\end{aligned}
$$

then $H_{n} \neq 0, I_{n} \neq 0$, and

$$
\begin{aligned}
& \alpha_{n m}=\frac{h_{n}^{(2)}(k \tilde{\rho})}{H_{n} \sqrt{n(n+1)}} a_{n m}, \quad \alpha_{n m}^{\prime}=-\frac{h_{n}^{(1)}(k \tilde{\rho})}{H_{n} \sqrt{n(n+1)}} a_{n m}, \\
& \beta_{n m}=\frac{z_{n}^{(2)}(k \tilde{\rho}) R}{I_{n} \sqrt{n(n+1)}} b_{n m}, \quad \beta_{n m}^{\prime}=-\frac{z_{n}^{(1)}(k \tilde{\rho}) R}{I_{n} \sqrt{n(n+1)}} b_{n m} .
\end{aligned}
$$

Since $\tilde{\mathbf{N}}_{n}^{m}=\frac{1}{1 k} \tilde{\nabla} \times \mathbf{M}_{n}^{m}, \hat{\mathbf{N}}_{n}^{m}=\frac{1}{1 k} \tilde{\nabla} \times \hat{\mathbf{M}}_{n}^{m}, \tilde{\mathbf{M}}_{n}^{m}=-\frac{1}{1 k} \nabla \times \tilde{\mathbf{N}}_{n}^{m}, \hat{\mathbf{M}}_{n}^{m}=-\frac{1}{1 k} \nabla \times \hat{\mathbf{N}}_{n}^{m}$, we have

$$
\begin{aligned}
& \hat{G}_{e}\left(\hat{\mathbf{x}} \times\left.\mathbf{\Phi}\right|_{\Gamma_{R}}\right) \\
= & \frac{1}{1 k} \hat{\mathbf{x}} \times\left.(\nabla \times \mathbf{w})\right|_{\Gamma_{R}} \\
= & \hat{\mathbf{x}} \times\left.\sum_{n=1}^{\infty} \sum_{m=-n}^{n}\left(\alpha_{n m} \tilde{\mathbf{N}}_{n}^{m}-\beta_{n m} \tilde{\mathbf{M}}_{n}^{m}+\alpha_{n m}^{\prime} \hat{\mathbf{N}}_{n}^{m}-\beta_{n m}^{\prime} \hat{\mathbf{M}}_{n}^{m}\right)\right|_{\Gamma_{R}} .
\end{aligned}
$$

By (3.5)-(3.6) and similar identities for $\hat{\mathbf{M}}_{n}^{m}, \hat{\mathbf{N}}_{n}^{m}$, we then get

$$
\begin{aligned}
& \hat{G}_{e}\left(\hat{\mathbf{x}} \times\left.\mathbf{\Phi}\right|_{\Gamma_{R}}\right) \\
&= \sum_{n=1}^{\infty} \sum_{m=-n}^{n} \frac{1}{1 k R} \sqrt{n(n+1)}\left(\alpha_{n m} z_{n}^{(1)}(k R)+\alpha_{n m}^{\prime} z_{n}^{(2)}(k R)\right) \mathbf{V}_{n}^{m} \\
& \quad+\sqrt{n(n+1)}\left(-\beta_{n m} h_{n}^{(1)}(k R)-\beta_{n m}^{\prime} h_{n}^{(2)}(k R)\right) \mathbf{U}_{n}^{m} \\
&=\sum_{n=1}^{\infty} \sum_{m=-n}^{n} \frac{1}{1 k R} \gamma_{n} a_{n m} \mathbf{V}_{n}^{m}-R \eta_{n} b_{n m} \mathbf{U}_{n}^{m},
\end{aligned}
$$

where $\gamma_{n}=\left(h_{n}^{(2)}(k \tilde{\rho}) z_{n}^{(1)}(k R)-h_{n}^{(1)}(k \tilde{\rho}) z_{n}^{(2)}(k R)\right) / H_{n}$ and $\eta_{n}=\left(z_{n}^{(2)}(k \tilde{\rho}) h_{n}^{(1)}(k R)-\right.$ $\left.z_{n}^{(1)}(k \tilde{\rho}) h_{n}^{(2)}(k R)\right) / I_{n}$. 
Now suppose that $\hat{\mathbf{x}} \times\left.\mathbf{\Psi}\right|_{\Gamma_{R}}=\sum_{n=1}^{\infty} \sum_{m=-n}^{n} a_{n m}^{\prime} \mathbf{U}_{n}^{m}+b_{n m}^{\prime} \mathbf{V}_{n}^{m}$; then

$$
\begin{aligned}
(\hat{\mathbf{x}} \times \mathbf{\Psi}) \times\left.\hat{\mathbf{x}}\right|_{\Gamma_{R}} & =\sum_{n=1}^{\infty} \sum_{m=-n}^{n} a_{n m}^{\prime} \mathbf{U}_{n}^{m} \times \hat{\mathbf{x}}+b_{n m}^{\prime} \mathbf{V}_{n}^{m} \times \hat{\mathbf{x}} \\
& =\sum_{n=1}^{\infty} \sum_{m=-n}^{n}-a_{n m}^{\prime} \mathbf{V}_{n}^{m}-b_{n m}^{\prime} \mathbf{U}_{n}^{m}
\end{aligned}
$$

Therefore

$$
\begin{aligned}
\left\langle\hat{G}_{e}(\hat{\mathbf{x}} \times \mathbf{\Phi}),(\hat{\mathbf{x}} \times \mathbf{\Psi}) \times \hat{\mathbf{x}}\right\rangle_{\Gamma_{R}} & =\left\langle\hat{G}_{e}(\hat{\mathbf{x}} \times \mathbf{\Phi}), \hat{\mathbf{x}} \times \mathbf{\Psi} \times \hat{\mathbf{x}}\right\rangle_{\Gamma_{R}} \\
& =\sum_{n=1}^{\infty} \sum_{m=-n}^{n}-\frac{1}{1 k R} \gamma_{n} a_{n m} \bar{a}_{n m}^{\prime}+R \eta_{n} b_{n m} \bar{b}_{n m}^{\prime} .
\end{aligned}
$$

This completes the proof.

Lemma 5.2 (Error representational formula). For any $\mathbf{\Phi} \in \mathbf{H}\left(\mathbf{c u r l} ; \Omega_{R}\right)$, which is extended to be a function $\hat{\boldsymbol{\Phi}}$ in $\mathbf{H}\left(\mathbf{c u r l} ; \Omega_{\rho}\right)$ according to (5.2)-(5.3), and $\mathbf{\Phi}_{h} \in \stackrel{\circ}{\mathbf{U}}_{h}$, we have

$$
a\left(\mathbf{E}-\mathbf{E}_{h}, \boldsymbol{\Phi}\right)=-b\left(\mathbf{E}_{h}, \hat{\mathbf{\Phi}}-\boldsymbol{\Phi}_{h}\right)+1 k\left\langle\left(\hat{G}_{e}-G_{e}\right)\left(\hat{\mathbf{x}} \times \mathbf{E}_{h}\right),(\hat{\mathbf{x}} \times \mathbf{\Phi}) \times \hat{\mathbf{x}}\right\rangle_{\Gamma_{R}} .
$$

Proof. By (2.13) and the definition of the sesquilinear forms $a(\cdot, \cdot)$ and $b(\cdot, \cdot)$, we have

$$
\begin{aligned}
a\left(\mathbf{E}-\mathbf{E}_{h}, \boldsymbol{\Phi}\right)= & -\int_{\Omega_{R}}\left(\nabla \times \mathbf{E}_{h} \cdot \nabla \times \overline{\mathbf{\Phi}}-k^{2} \mathbf{E}_{h} \cdot \overline{\mathbf{\Phi}}\right) d \mathbf{x} \\
& -1 k\left\langle G_{e}\left(\hat{\mathbf{x}} \times \mathbf{E}_{h}\right),(\hat{\mathbf{x}} \times \mathbf{\Phi}) \times \hat{\mathbf{x}}\right\rangle_{\Gamma_{R}} \\
= & -b\left(\mathbf{E}_{h}, \hat{\mathbf{\Phi}}\right)+\int_{\Omega^{\mathrm{PML}}}\left(B A \nabla \times \mathbf{E}_{h} \cdot \nabla \times \overline{\hat{\mathbf{\Phi}}}-k^{2}(B A)^{-1} \mathbf{E}_{h} \cdot \overline{\hat{\mathbf{\Phi}}}\right) \\
& -1 k\left\langle G_{e}\left(\hat{\mathbf{x}} \times \mathbf{E}_{h}\right),(\hat{\mathbf{x}} \times \mathbf{\Phi}) \times \hat{\mathbf{x}}\right\rangle_{\Gamma_{R}} .
\end{aligned}
$$

Integrate by parts and use (5.2) to obtain $\nabla \times B A \nabla \times \overline{\hat{\boldsymbol{\Phi}}}-k^{2}(B A)^{-1} \overline{\hat{\boldsymbol{\Phi}}}=0$ in $\Omega^{\mathrm{PML}}$, we have

$$
\begin{aligned}
a\left(\mathbf{E}-\mathbf{E}_{h}, \mathbf{\Phi}\right)= & -b\left(\mathbf{E}_{h}, \hat{\mathbf{\Phi}}\right)+\left\langle\mathbf{n} \times \mathbf{E}_{h}, \mathbf{n} \times(\overline{B A} \nabla \times \hat{\boldsymbol{\Phi}}) \times \mathbf{n}\right\rangle_{\Gamma_{R} \cup \Gamma_{\rho}} \\
& -1 k\left\langle G_{e}\left(\hat{\mathbf{x}} \times \mathbf{E}_{h}\right),(\hat{\mathbf{x}} \times \mathbf{\Phi}) \times \hat{\mathbf{x}}\right\rangle_{\Gamma_{R}} .
\end{aligned}
$$

Since $\mathbf{n} \times \mathbf{E}_{h}=0$ on $\Gamma_{\rho}$ and $\mathbf{n}=-\hat{\mathbf{x}}$ on $\Gamma_{R}$ for the domain $\Omega^{\mathrm{PML}}$, we get

$$
\begin{aligned}
& a\left(\mathbf{E}-\mathbf{E}_{h}, \boldsymbol{\Phi}\right) \\
= & -b\left(\mathbf{E}_{h}, \hat{\mathbf{\Phi}}\right)-\left\langle\hat{\mathbf{x}} \times \mathbf{E}_{h}, \hat{\mathbf{x}} \times(\nabla \times \hat{\mathbf{\Phi}}) \times \hat{\mathbf{x}}\right\rangle_{\Gamma_{R}}-1 k\left\langle G_{e}\left(\hat{\mathbf{x}} \times \mathbf{E}_{h}\right),(\hat{\mathbf{x}} \times \mathbf{\Phi}) \times \hat{\mathbf{x}}\right\rangle_{\Gamma_{R}} .
\end{aligned}
$$

By (2.15) and (5.2)-(15.3), we know that $\hat{G}_{e}(\hat{\mathbf{x}} \times \overline{\mathbf{\Phi}})=\frac{1}{1 k} \hat{\mathbf{x}} \times(\nabla \times \overline{\hat{\mathbf{\Phi}}})$. Thus

$$
\begin{aligned}
& a\left(\mathbf{E}-\mathbf{E}_{h}, \mathbf{\Phi}\right) \\
= & -b\left(\mathbf{E}_{h}, \hat{\mathbf{\Phi}}\right)+1 k\left\langle\hat{\mathbf{x}} \times \mathbf{E}_{h}, \hat{G}_{e}(\hat{\mathbf{x}} \times \mathbf{\Phi}) \times \hat{\mathbf{x}}\right\rangle_{\Gamma_{R}}-1 k\left\langle G_{e}\left(\hat{\mathbf{x}} \times \mathbf{E}_{h}\right),(\hat{\mathbf{x}} \times \mathbf{\Phi}) \times \hat{\mathbf{x}}\right\rangle_{\Gamma_{R}} \\
= & -b\left(\mathbf{E}_{h}, \hat{\mathbf{\Phi}}\right)+1 k\left\langle\hat{G}_{e}(\hat{\mathbf{x}} \times \overline{\mathbf{\Phi}}),\left(\hat{\mathbf{x}} \times \overline{\mathbf{E}}_{h}\right) \times \hat{\mathbf{x}}\right\rangle_{\Gamma_{R}}-1 k\left\langle G_{e}\left(\hat{\mathbf{x}} \times \mathbf{E}_{h}\right),(\hat{\mathbf{x}} \times \mathbf{\Phi}) \times \hat{\mathbf{x}}\right\rangle_{\Gamma_{R}} \\
= & -b\left(\mathbf{E}_{h}, \hat{\mathbf{\Phi}}\right)+1 k\left\langle\left(\hat{G}_{e}-G_{e}\right)\left(\hat{\mathbf{x}} \times \mathbf{E}_{h}\right),(\hat{\mathbf{x}} \times \mathbf{\Phi}) \times \hat{\mathbf{x}}\right\rangle_{\Gamma_{R}},
\end{aligned}
$$

where in the last equality we have used Lemma [5.1. This completes the proof by using (4.3). 
5.2. Stability estimates for the extension. We start by proving two estimates for the PML extension in (2.9).

Lemma 5.3. There exists a constant $C>0$ independent of $k, R, \rho$ and $\sigma_{0}$ such that for any $\lambda \in \mathbf{H}^{-1 / 2}\left(\operatorname{Div} ; \Gamma_{\mathrm{R}}\right)$,

$$
\|\nabla \times B \mathbb{E}(\lambda)\|_{\mathbf{L}^{2}\left(\Omega^{\mathrm{PML}}\right)} \leq C R^{1 / 2}\left|\alpha_{0}\right|^{2}(1+k R)^{3}\|\lambda\|_{\mathbf{H}^{-1 / 2}\left(\operatorname{Div} ; \Gamma_{\mathrm{R}}\right)} .
$$

Proof. Let $\lambda=\sum_{n=1}^{\infty} \sum_{m=-n}^{n} a_{m n} \mathbf{U}_{n}^{m}+b_{m n} \mathbf{V}_{n}^{m}$ and denote $\tilde{\boldsymbol{\Phi}}=\mathbb{E}(\lambda)$. By Lemma 3.4 and (2.7)-(2.8), we obtain

$$
\begin{gathered}
\frac{1}{1 k} \tilde{\nabla} \times \tilde{\boldsymbol{\Phi}}=\sum_{n=1}^{\infty} \sum_{m=-n}^{n}\left(\frac{a_{n m}}{1 k \tilde{r}} \frac{z_{n}^{(1)}(k \tilde{r})}{h_{n}^{(1)}(k R)} \mathbf{U}_{n}^{m}+1 k R b_{m n} \frac{h_{n}^{(1)}(k \tilde{r})}{z_{n}^{(1)}(k R)} \mathbf{V}_{n}^{m}\right. \\
\left.+\frac{a_{n m}}{1 k \tilde{r}} \frac{h_{n}^{(1)}(k \tilde{r})}{h_{n}^{(1)}(k R)} \sqrt{n(n+1)} Y_{n}^{m} \hat{\mathbf{x}}\right)
\end{gathered}
$$

which yields

$$
\begin{aligned}
& \int_{\Omega^{\mathrm{PML}}} \frac{1}{k^{2} r^{2}}|\tilde{\nabla} \times \tilde{\boldsymbol{\Phi}}|^{2} d \mathbf{x} \\
= & \sum_{n=1}^{\infty} \sum_{m=-n}^{n} \int_{R}^{\rho} \frac{\left|a_{n m}\right|^{2}}{k^{2}|\tilde{r}|^{2}}\left(\left|\frac{z_{n}^{(1)}(k \tilde{r})}{h_{n}^{(1)}(k R)}\right|^{2}+n(n+1)\left|\frac{h_{n}^{(1)}(k \tilde{r})}{h_{n}^{(1)}(k R)}\right|^{2}\right) d r \\
& +\sum_{n=1}^{\infty} \sum_{m=-n}^{n} \int_{R}^{\rho} k^{2} R^{2}\left|\frac{h_{n}^{(1)}(k \tilde{r})}{z_{n}^{(1)}(k R)}\right|^{2}\left|b_{n m}\right|^{2} d r .
\end{aligned}
$$

By the identity $h_{n}^{(1) \prime}(z)=-\frac{n+1}{z} h_{n}^{(1)}(z)+h_{n-1}^{(1)}(z)$, we get

$$
\begin{aligned}
\int_{R}^{\rho} \frac{1}{k^{2}|\tilde{r}|^{2}}\left|\frac{z_{n}^{(1)}(k \tilde{r})}{h_{n}^{(1)}(k R)}\right|^{2} d r & =\int_{R}^{\rho} \frac{1}{k^{2}|\tilde{r}|^{2}}\left|\frac{-n h_{n}^{(1)}(k \tilde{r})+k \tilde{r} h_{n-1}^{(1)}(k \tilde{r})}{h_{n}^{(1)}(k R)}\right|^{2} d r \\
& \leq \frac{n^{2}}{k^{2} R^{2}} \int_{R}^{\infty}\left|\frac{h_{n}^{(1)}(k \tilde{r})}{h_{n}^{(1)}(k R)}\right|^{2} d r+\int_{R}^{\rho}\left|\frac{h_{n-1}^{(1)}(k \tilde{r})}{h_{n}^{(1)}(k R)}\right|^{2} d r
\end{aligned}
$$

To proceed, we recall the following estimate due to Nédeléc [20, (2.6.59)]:

$$
\int_{1}^{\infty}\left|\frac{h_{n}^{(1)}(k r)}{h_{n}^{(1)}(k)}\right|^{2} d r \leq \frac{6 k^{2}+n+1}{6 k^{2}+(2 n+1)(n+1)}, \quad \forall n \geq 1 .
$$

Thus

$$
\begin{aligned}
\int_{R}^{\infty}\left|\frac{h_{n}^{(1)}(k r)}{h_{n}^{(1)}(k R)}\right|^{2} d r & =R \int_{1}^{\infty}\left|\frac{h_{n}^{(1)}(k R r)}{h_{n}^{(1)}(k R)}\right|^{2} d r \\
& \leq R \cdot \frac{6 k^{2} R^{2}+n+1}{6 k^{2} R^{2}+(2 n+1)(n+1)} \\
& \leq \frac{R}{2 n+1}\left(1+\frac{6 k^{2} R^{2}}{n+1}\right) .
\end{aligned}
$$


Therefore

$$
\begin{aligned}
\int_{R}^{\rho} \frac{1}{k^{2}|\tilde{r}|^{2}}\left|\frac{z_{n}^{(1)}(k \tilde{r})}{h_{n}^{(1)}(k R)}\right|^{2} d r & \leq \frac{n^{2}}{k^{2} R^{2}} \cdot \frac{R}{2 n+1}\left(1+\frac{6 k^{2} R^{2}}{n+1}\right)+(\rho-R) \\
& \leq C R \sqrt{n(n+1)}\left(1+\frac{1}{k^{2} R^{2}}\right)
\end{aligned}
$$

where we have used (5.7), (3.7) and the inequality $\left|h_{n-1}^{(1)}(\Theta)\right| \leq\left|h_{n}^{(1)}(\Theta)\right|$ for any $\Theta>0$ to conclude that $\left|h_{n-1}^{(1)}(k \tilde{r})\right| \leq\left|h_{n}^{(1)}(k R)\right|$. Similarly

$$
n(n+1) \int_{R}^{\rho} \frac{1}{k^{2}|\tilde{r}|^{2}}\left|\frac{h_{n}^{(1)}(k \tilde{r})}{h_{n}^{(1)}(k R)}\right|^{2} d r \leq C R \sqrt{n(n+1)}\left(1+\frac{1}{k^{2} R^{2}}\right) .
$$

By (3.9) we have $\left|\delta_{n}(k R)\right|^{-1} \leq C$, thus

$$
\begin{aligned}
\int_{R}^{\rho} k^{2} R^{2}\left|\frac{h_{n}^{(1)}(k \tilde{r})}{z_{n}^{(1)}(k R)}\right|^{2} d r & =k^{2} R^{2}\left|\delta_{n}(k R)\right|^{-2} \int_{R}^{\rho}\left|\frac{h_{n}^{(1)}(k \tilde{r})}{h_{n}^{(1)}(k R)}\right|^{2} d r \\
& \leq C k^{2} R^{2} \int_{R}^{\rho}\left|\frac{h_{n}^{(1)}(k \tilde{r})}{h_{n}^{(1)}(k R)}\right|^{2} d r \\
& \leq C k^{2} R^{2} \cdot \frac{R}{2 n+1}\left(1+\frac{6 k^{2} R^{2}}{n+1}\right) \\
& \leq C k^{2} R^{2}\left(1+k^{2} R^{2}\right) \frac{R}{\sqrt{n(n+1)}} .
\end{aligned}
$$

Substituting (5.8)-(5.9) into (5.6) we get

$$
\begin{aligned}
& \int_{\Omega^{\mathrm{PML}}} \frac{1}{r^{2}}|\tilde{\nabla} \times \tilde{\boldsymbol{\Phi}}|^{2} d \mathbf{x} \\
\leq & C R\left(1+\frac{1}{k^{2} R^{2}}+k^{4} R^{4}\right) \sum_{n=1}^{\infty} \sum_{m=-n}^{n} \sqrt{n(n+1)}\left|a_{n m}\right|^{2}+\frac{1}{\sqrt{n(n+1)}}\left|b_{n m}\right|^{2} \\
= & C R\left(1+\frac{1}{k^{2} R^{2}}+k^{4} R^{4}\right)\|\lambda\|_{\mathbf{H}^{-1 / 2}\left(\text { Div; } \Gamma_{\mathrm{R}}\right)}^{2} .
\end{aligned}
$$

Hence

$$
\|\tilde{\nabla} \times \tilde{\boldsymbol{\Phi}}\|_{\mathbf{L}^{2}\left(\Omega^{\mathrm{PML}}\right)} \leq C R^{1 / 2}(1+k R)^{3}\|\lambda\|_{\mathbf{H}^{-1 / 2}\left(\operatorname{Div} ; \Gamma_{\mathrm{R}}\right)} .
$$

This completes the proof since $\tilde{\nabla} \times \tilde{\boldsymbol{\Phi}}=A \nabla \times B \tilde{\boldsymbol{\Phi}}, A=\operatorname{diag}\left(\beta^{-2}, \alpha^{-1} \beta^{-1}, \alpha^{-1} \beta^{-1}\right)$, and $\left|\beta^{-2}\right| \geq\left|\alpha_{0}\right|^{-2},\left|\alpha^{-1} \beta^{-1}\right| \geq\left|\alpha_{0}\right|^{-2}$.

Lemma 5.4. There exists a constant $C>0$ independent of $k, R, \rho$, and $\sigma_{0}$ such that for any $\lambda \in \mathbf{H}^{-1 / 2}\left(\operatorname{Div} ; \Gamma_{\mathrm{R}}\right)$,

$$
\|B \mathbb{E}(\lambda)\|_{\mathbf{L}^{2}\left(\Omega^{\mathrm{PML}}\right)} \leq C R^{3 / 2}\left|\alpha_{0}\right|(1+k R)^{2}\|\lambda\|_{\mathbf{H}^{-1 / 2}\left(\operatorname{Div} ; \Gamma_{\mathrm{R}}\right)} .
$$

Proof. Let $\lambda=\sum_{n=1}^{\infty} \sum_{m=-n}^{n} a_{m n} \mathbf{U}_{n}^{m}+b_{m n} \mathbf{V}_{n}^{m}$ and denote $\tilde{\boldsymbol{\Phi}}=\mathbb{E}(\lambda)$. From (2.9) and (2.7)-(2.8), we have

$$
\begin{gathered}
\tilde{\boldsymbol{\Phi}}=\sum_{n=1}^{\infty} \sum_{m=-n}^{n}\left(\frac{R}{\tilde{r}} \frac{z_{n}^{(1)}(k \tilde{r})}{z_{n}^{(1)}(k R)} b_{n m} \mathbf{U}_{n}^{m}-\frac{h_{n}^{(1)}(k \tilde{r})}{h_{n}^{(1)}(k R)} a_{n m} \mathbf{V}_{n}^{m}\right. \\
\left.+\frac{R}{\tilde{r}} \sqrt{n(n+1)} \frac{h_{n}^{(1)}(k \tilde{r})}{z_{n}^{(1)}(k R)} b_{m n} Y_{n}^{m} \hat{\mathbf{x}}\right) .
\end{gathered}
$$


Thus

$$
\begin{aligned}
\int_{\Omega^{\mathrm{PML}}} \frac{1}{r^{2}}|\tilde{\mathbf{\Phi}}|^{2} d \mathbf{x}= & \sum_{n=1}^{\infty} \sum_{m=-n}^{n} \int_{R}^{\rho}\left|\frac{h_{n}^{(1)}(k \tilde{r})}{h_{n}^{(1)}(k R)}\right|^{2}\left|a_{n m}\right|^{2} d r \\
& +\int_{R}^{\rho}\left|\frac{R}{\tilde{r}}\right|^{2}\left(\left|\frac{z_{n}^{(1)}(k \tilde{r})}{z_{n}^{(1)}(k R)}\right|^{2}+n(n+1)\left|\frac{h_{n}^{(1)}(k \tilde{r})}{z_{n}^{(1)}(k R)}\right|^{2}\right)\left|b_{n m}\right|^{2} d r .
\end{aligned}
$$

Since $\left|h_{n}^{(1)}(k \tilde{r})\right| \leq\left|h_{n}^{(1)}(k R)\right|$ by Lemma 3.2, we have

$$
\int_{R}^{\rho}\left|\frac{h_{n}^{(1)}(k \tilde{r})}{h_{n}^{(1)}(k R)}\right|^{2} d r \leq \rho-R \leq C R .
$$

By (3.9) we have $\left|\delta_{n}(k R)\right|^{-1} \leq C n^{-1}(1+k R)$. Thus by (5.8), we have

$$
\begin{aligned}
& \int_{R}^{\rho}\left|\frac{R}{\tilde{r}}\right|^{2}\left|\frac{z_{n}^{(1)}(k \tilde{r})}{z_{n}^{(1)}(k R)}\right|^{2} d r \\
= & k^{2} R^{2} \int_{R}^{\rho} \frac{1}{k^{2}|\tilde{r}|^{2}}\left|\frac{z_{n}^{(1)}(k \tilde{r})}{h_{n}^{(1)} k R}\right|^{2} d r \cdot\left|\delta_{n}(k R)\right|^{-2} \\
\leq & k^{2} R^{2} \cdot C R \sqrt{n(n+1)}\left(1+\frac{1}{k^{2} R^{2}}\right) \cdot C \frac{(1+k R)^{2}}{n^{2}} \\
\leq & C R \frac{1}{\sqrt{n(n+1)}}(1+k R)^{4} .
\end{aligned}
$$

Finally, by (5.8) we get

$$
\begin{aligned}
& n(n+1) \int_{R}^{\rho} \frac{R^{2}}{|\tilde{r}|^{2}}\left|\frac{h_{n}^{(1)}(k \tilde{r})}{z_{n}^{(1)}(k R)}\right|^{2} d r \\
= & k^{2} R^{2} \cdot n(n+1) \cdot \int_{R}^{\rho} \frac{1}{k^{2}|\tilde{r}|^{2}}\left|\frac{h_{n}^{(1)}(k \tilde{r})}{h_{n}^{(1)}(k R)}\right|^{2} d r \cdot\left|\delta_{n}(k R)\right|^{-2} \\
\leq & k^{2} R^{2} \cdot C R \sqrt{n(n+1)}\left(1+\frac{1}{k^{2} R^{2}}\right) \cdot C \frac{(1+k R)^{2}}{n^{2}} \\
\leq & C R \frac{1}{\sqrt{n(n+1)}}(1+k R)^{4} .
\end{aligned}
$$

Substituting (5.10)-(5.12) into (5.10), we obtain

$$
\begin{aligned}
\int_{\Omega^{\mathrm{PML}}} \frac{1}{r^{2}}|\tilde{\mathbf{\Phi}}|^{2} d \mathbf{x} & \leq C R(1+k R)^{4} \sum_{n=1}^{\infty} \sum_{m=-n}^{n}\left|a_{n m}\right|^{2}+\frac{1}{\sqrt{n(n+1)}}\left|b_{n m}\right|^{2} \\
& \leq C R(1+k R)^{4}\|\lambda\|_{\mathbf{H}^{-1 / 2}(\text { Div; } ; \mathrm{R})}^{2},
\end{aligned}
$$

which implies

$$
\|\tilde{\boldsymbol{\Phi}}\|_{\mathbf{L}^{2}\left(\Omega^{\mathrm{PML}}\right)} \leq C R^{3 / 2}(1+k R)^{2}\|\lambda\|_{\mathbf{H}^{-1 / 2}\left(\mathrm{Div} ; \Gamma_{\mathrm{R}}\right)} .
$$

This completes the proof by using the fact that $B=\operatorname{diag}(\alpha, \beta, \beta)$ and $|\alpha| \leq$ $\left|\alpha_{0}\right|,|\beta| \leq\left|\alpha_{0}\right|$.

The following result on the extension $\hat{\boldsymbol{\Phi}}$ is the main objective of this subsection. 
Lemma 5.5. Let $\hat{\mathbf{\Phi}}$ be the extension of $\mathbf{\Phi} \in \mathbf{H}\left(\mathbf{c u r l} ; \Omega_{R}\right)$ according to (5.2) $-(\sqrt{5.3})$. Then we have

$$
\|\hat{\mathbf{\Phi}}\|_{\mathbf{c u r l} ; \Omega^{\mathrm{PML}}} \leq C \hat{C}^{-1}\left|\alpha_{0}\right|^{3} R^{1 / 2}(1+k R)^{3}\|\hat{\mathbf{x}} \times \mathbf{\Phi}\|_{\mathbf{H}^{-1 / 2}\left(\operatorname{Div} ; \Gamma_{\mathrm{R}}\right)} .
$$

Proof. Let $\tilde{\boldsymbol{\Phi}}=\mathbb{E}\left(\hat{\mathbf{x}} \times\left.\overline{\boldsymbol{\Phi}}\right|_{\Gamma_{R}}\right)$ be the PML extension of $\hat{\mathbf{x}} \times\left.\overline{\boldsymbol{\Phi}}\right|_{\Gamma_{R}}$. Then $\tilde{\boldsymbol{\Phi}}$ satisfies

$$
\begin{aligned}
\nabla \times B A \nabla \times \tilde{\boldsymbol{\Phi}}-k^{2}(B A)^{-1} \tilde{\boldsymbol{\Phi}} & =0 \quad \text { in } \mathbb{R}^{3} \backslash \bar{B}_{R}, \\
\hat{\mathbf{x}} \times \tilde{\boldsymbol{\Phi}} & =\hat{\mathbf{x}} \times \overline{\mathbf{\Phi}} \text { on } \Gamma_{R} .
\end{aligned}
$$

Let $\mathbf{w}=\hat{\mathbf{\Phi}}-B \overline{\tilde{\mathbf{\Phi}}} ;$ then $\mathbf{w}$ satisfies

$$
\begin{aligned}
& \nabla \times B A \nabla \times \mathbf{w}-k^{2}(B A)^{-1} \mathbf{w}=0 \quad \text { in } \Omega^{\mathrm{PML}}, \\
& \hat{\mathbf{x}} \times \mathbf{w}=0 \text { on } \Gamma_{R}, \quad \hat{\mathbf{x}} \times \mathbf{w}=-B \mathbb{P}\left(\hat{\mathbf{x}} \times\left.\overline{\mathbf{\Phi}}\right|_{\Gamma_{R}}\right) \text { on } \Gamma_{\rho} .
\end{aligned}
$$

By Lemma 3.6 and (3.15)

$$
\begin{aligned}
\|\mathbf{w}\|_{\text {curl } ; \Omega^{\mathrm{PML}}} & \leq C \hat{C}^{-1}\left|\alpha_{0}\right|^{2}\left\|B \mathbb{P}\left(\hat{\mathbf{x}} \times\left.\overline{\mathbf{\Phi}}\right|_{\Gamma_{R}}\right)\right\|_{\mathbf{H}^{-1 / 2}\left(\operatorname{Div} ; \Gamma_{\rho}\right)} \\
& \leq C \hat{C}^{-1}(1+k R)\left|\alpha_{0}\right|^{3}\|\hat{\mathbf{x}} \times \mathbf{\Phi}\|_{\mathbf{H}^{-1 / 2}\left(\operatorname{Div} ; \Gamma_{\mathrm{R}}\right)} .
\end{aligned}
$$

By Lemmas 5.35.5, we have

$$
\|B \tilde{\boldsymbol{\Phi}}\|_{\mathbf{c u r l} ; \Omega^{\mathrm{PML}}} \leq C R^{1 / 2}(1+k R)^{3}\left|\alpha_{0}\right|^{3}\|\hat{\mathbf{x}} \times \mathbf{\Phi}\|_{\mathbf{H}^{-1 / 2}\left(\operatorname{Div} ; \Gamma_{\mathrm{R}}\right)} .
$$

This completes the proof by using the triangle inequality.

5.3. Proof of Theorem 4.1. Our starting point is (5.1). To estimate the second term in (5.1), for any $\boldsymbol{\Phi} \in \mathbf{H}\left(\mathbf{c u r l} ; \Omega_{R}\right)$ such that $\mathbf{n} \times \mathbf{\Phi}=0$ on $\Gamma_{D}$, we denote by $\hat{\boldsymbol{\Phi}}$ its extension to $\Omega^{\mathrm{PML}}$ according to (5.2)-(5.3). Thus $\hat{\boldsymbol{\Phi}} \in \mathbf{H}_{0}\left(\operatorname{curl} ; \Omega_{\rho}\right)$. By Lemma 4.2, there exists $\boldsymbol{\Psi} \in \mathbf{H}_{0}\left(\mathbf{c u r l} ; \Omega_{\rho}\right) \cap H^{1}\left(\Omega_{\rho}\right)^{3}$ and $\varphi \in H_{0}^{1}\left(\Omega_{\rho}\right)$ such that $\hat{\boldsymbol{\Phi}}=\boldsymbol{\Psi}+\nabla \varphi$, and

$$
\|\varphi\|_{H^{1}\left(\Omega_{\rho}\right)}+\|\boldsymbol{\Psi}\|_{\mathbf{H}^{1}\left(\Omega_{\rho}\right)} \leq C\|\hat{\mathbf{\Phi}}\|_{\mathbf{H}\left(\operatorname{curl} ; \Omega_{\rho}\right)} .
$$

By Lemma 5.5 and the trace inequality for $\mathbf{H}\left(\mathbf{c u r l} ; \Omega_{R}\right)$, we then have

$$
\|\varphi\|_{H^{1}\left(\Omega_{\rho}\right)}+\|\Psi\|_{\mathbf{H}^{1}\left(\Omega_{\rho}\right)} \leq C \hat{C}^{-1} R^{1 / 2}\left|\alpha_{0}\right|^{3}(1+k R)^{3}\|\mathbf{\Phi}\|_{\mathbf{H}\left(\mathbf{c u r l} ; \Omega_{R}\right)} .
$$

Let $\mathbf{\Phi}_{h}=\nabla r_{h} \varphi+\pi_{h} \boldsymbol{\Psi}$, where $r_{h}: H_{0}^{1}\left(\Omega_{\rho}\right) \rightarrow \stackrel{\circ}{V}_{h}$ and $\pi_{h}: H^{1}\left(\Omega_{\rho}\right)^{3} \cap \mathbf{H}_{0}\left(\mathbf{c u r l} ; \Omega_{\rho}\right)$ $\rightarrow \stackrel{\circ}{\mathbf{U}}_{h}$ are the interpolation operators defined at the end of $\S 4$. By the error representation formula in Lemma 5.2 , we have

$$
\begin{aligned}
& a\left(\mathbf{E}-\mathbf{E}_{h}, \mathbf{\Phi}\right) \\
= & -b\left(\mathbf{E}_{h}, \mathbf{\Psi}+\nabla \varphi-\left(\pi_{h} \mathbf{\Psi}+\nabla r_{h} \varphi\right)\right)+1 k\left\langle\left(\hat{G}_{e}-G_{e}\right)\left(\hat{\mathbf{x}} \times \mathbf{E}_{h}\right),(\hat{\mathbf{x}} \times \mathbf{\Phi}) \times \hat{\mathbf{x}}\right\rangle_{\Gamma_{R}} \\
=\quad & -\int_{\Omega_{\rho}}\left(B A \nabla \times \mathbf{E}_{h} \cdot \nabla \times\left(\overline{\mathbf{\Psi}}-\pi_{h} \overline{\mathbf{\Psi}}\right)-k^{2}(B A)^{-1} \mathbf{E}_{h} \cdot\left(\overline{\mathbf{\Psi}}-\pi_{h} \overline{\mathbf{\Psi}}\right)\right) d \mathbf{x} \\
& +\int_{\Omega_{\rho}} k^{2}(B A)^{-1} \mathbf{E}_{h} \cdot \nabla\left(\bar{\varphi}-r_{h} \bar{\varphi}\right) d \mathbf{x} \\
& +1 k\left\langle\left(\hat{G}_{e}-G_{e}\right)\left(\hat{\mathbf{x}} \times \mathbf{E}_{h}\right),(\hat{\mathbf{x}} \times \mathbf{\Phi}) \times \hat{\mathbf{x}}\right\rangle_{\Gamma_{R}} \\
:= & \mathrm{I}+\mathrm{II}+\mathrm{III} .
\end{aligned}
$$


By using integration by parts, the estimates (4.4)-(4.5), and the standard argument in the a posteriori error analysis, we obtain

$$
\begin{aligned}
|\mathrm{I}+\mathrm{II}| & \leq C\left(\sum_{K \in \mathcal{M}_{h}} \eta_{K}^{2}\right)^{1 / 2}\left(\|\varphi\|_{H^{1}\left(\Omega_{\rho}\right)}+\|\boldsymbol{\Psi}\|_{\mathbf{H}^{1}\left(\Omega_{\rho}\right)}\right) \\
& \leq C \hat{C}^{-1} R^{1 / 2}\left|\alpha_{0}\right|^{3}(1+k R)^{3}\left(\sum_{K \in \mathcal{M}_{h}} \eta_{K}^{2}\right)^{1 / 2}\|\mathbf{\Phi}\|_{\mathbf{H}\left(\mathbf{c u r l} ; \Omega_{R}\right)},
\end{aligned}
$$

where we have used (5.13) in the last inequality. By Lemma 3.7 and trace inequality for $\mathbf{H}\left(\operatorname{curl} ; \Omega_{R}\right)$, we have

$|\mathrm{III}| \leq C \hat{C}^{-1}(1+k R)^{3}\left|\alpha_{0}\right|^{3} e^{-\operatorname{Im}(k \tilde{\rho})\left(1-\frac{R^{2}}{|\tilde{\rho}|^{2}}\right)^{1 / 2}}\left\|\hat{\mathbf{x}} \times \mathbf{E}_{h}\right\|_{\mathbf{H}^{-1 / 2}\left(\operatorname{Div} ; \Gamma_{\mathrm{R}}\right)}\|\boldsymbol{\Phi}\|_{\mathbf{H}\left(\mathbf{c u r l} ; \Omega_{R}\right)}$.

This completes the proof by (5.1).

\section{Numerical EXAMPles}

The implementation of the adaptive algorithm in this section is based on the adaptive finite element package ALBERT [22] and its adaptation to the edge element by Dr. Long Wang. The computation is carried out on an Origin 3800. We use the a posteriori error estimate in Theorem 4.1 to determine the PML parameters. According to the discussion in section 3, we choose the PML medium property as the power function and thus we need only to specify the thickness $\rho-R$ of the layer and the medium parameter $\sigma_{0}$. Recall from Theorem 4.1 that the a posteriori error estimate consists of two parts: the PML error and the finite element discretization error. In our implementation we first choose $\rho$ and $\sigma_{0}$ such that the exponentially decaying factor

$$
e^{-k \operatorname{Im}(\tilde{\rho})\left(1-\frac{R^{2}}{|\tilde{\rho}|^{2}}\right)^{1 / 2}} \leq 10^{-8}
$$

which makes the PML error negligible compared with the finite element discretization errors. Once the PML region and the medium property are fixed, we use the standard finite element adaptive strategy to modify the mesh according to the a posteriori error estimate (cf. e.g. 8]).

In the following we report two numerical examples to demonstrate the competitive behavior of the proposed algorithm.

Example 1. Let the scatterer $D$ be the unit ball and $k=1$. We consider the scattering problem whose exact solution is known:

$$
\mathbf{E}=\mathbf{M}_{1}^{0}(|\mathbf{x}|, \hat{\mathbf{x}})=\nabla \times\left\{\mathbf{x} h_{1}^{(1)}(|\mathbf{x}|) Y_{1}^{0}(\hat{\mathbf{x}})\right\} .
$$

Figure 6.1] shows the $\log N_{k}-\log \left\|\nabla \times\left(\mathbf{E}-\mathbf{E}_{k}\right)\right\|_{\mathbf{L}^{2}\left(\Omega_{R}\right)}$ curves, where $N_{k}$ is the number of edges of the mesh $\mathcal{M}_{k}$ and $\mathbf{E}_{k}$ is the finite element solution of (4.3) over the mesh $\mathcal{M}_{k}$. It indicates that the meshes and the associated numerical complexity are quasi-optimal: $\left\|\nabla \times\left(\mathbf{E}-\mathbf{E}_{k}\right)\right\|_{\mathbf{L}^{2}\left(\Omega_{R}\right)} \approx C N_{k}^{-1 / 3}$ is valid asymptotically.

One of the important quantities in the scattering problem is the far field

$$
\mathbf{E}_{\infty}(\hat{\mathbf{x}})=\frac{1 k}{4 \pi} \hat{\mathbf{x}} \times \int_{\Gamma_{D}}\left((\mathbf{n} \times \mathbf{E})(\mathbf{y})+\left(\mathbf{n} \times \frac{1}{1 k} \nabla \times \mathbf{E}\right)(\mathbf{y}) \times \hat{\mathbf{x}}\right) e^{-1 k \hat{\mathbf{x}} \cdot \mathbf{y}} d S_{\mathbf{y}} .
$$

Figures 6.2 shows the far fields for different choices of PML parameters $\rho$ and $\sigma_{0}$. We observe that our adaptive algorithm is robust with respect to the choice of the 


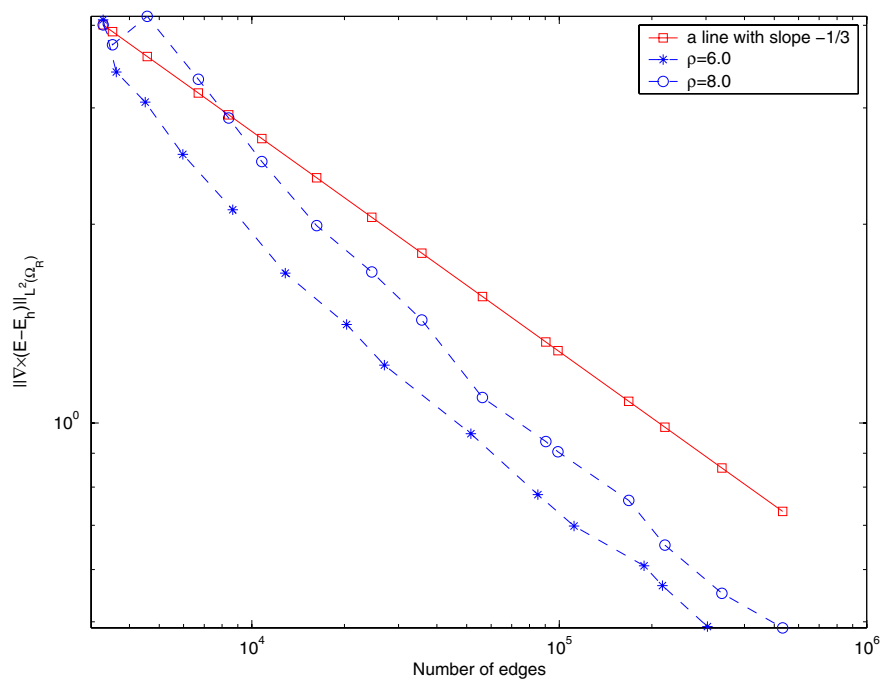

Figure 6.1. The quasi-optimality of the adaptive mesh refinements of the error $\left\|\nabla \times\left(\mathbf{E}-\mathbf{E}_{k}\right)\right\|_{\mathbf{L}^{2}\left(\Omega_{R}\right)}$ for Example 1 .

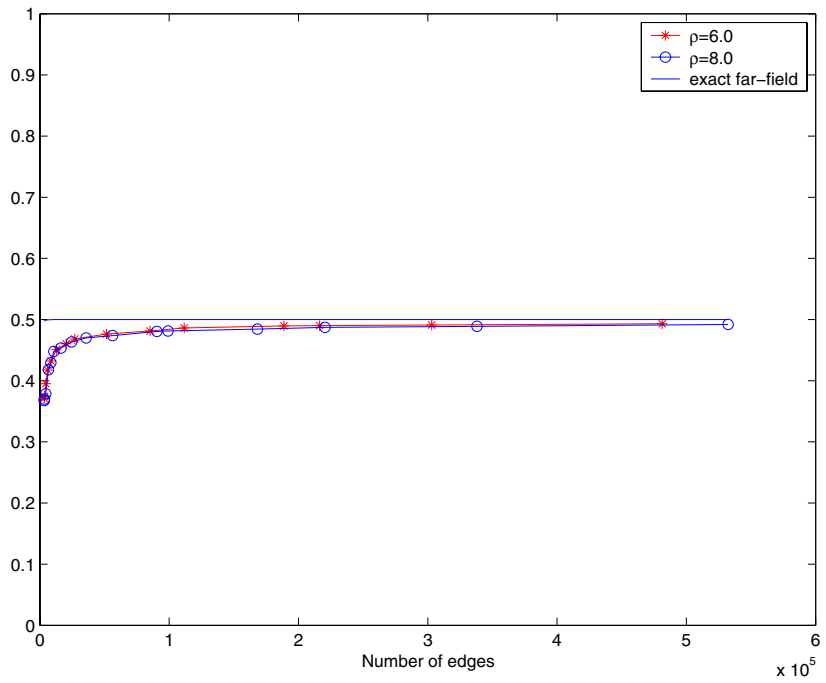

Figure 6.2. The module of the real part of the far fields in the direction $(1,0,0)$ for Example 1.

thickness of the PML layer: the far fields of the scattering solutions are insensitive to the choices of the PML parameters.

Example 2. This example concerns the scattering of the plane wave $\mathbf{E}^{i}=e^{1 x_{3}} \mathbf{e}_{1}$ from a perfectly conducting metal. The scatterer $D$ is shown in Figure 6.3.

In Figure 6.4 we show the mesh after 18 adaptive iterations when $\rho=2 R=$ 6 with 817078 edges. Figure 6.5 shows the $\log N_{k}-\log \mathcal{E}_{k}$ curves, where $\mathcal{E}_{k}=$ 


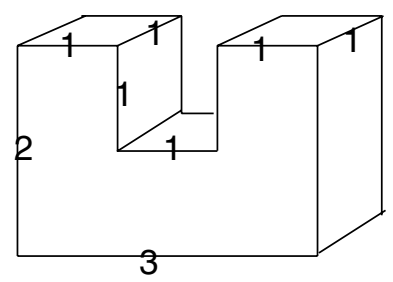

Figure 6.3. The U-shape scatterer for Example 2.

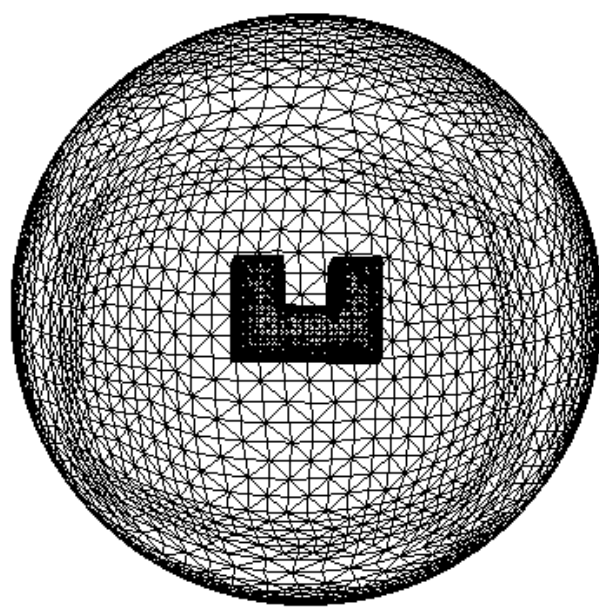

Figure 6.4. The mesh of 817078 edges after 18 adaptive refinements, $\rho=2 R=6$ for Example 2 .

$\left(\sum_{K \in \mathcal{M}_{k}} \eta_{K}^{2}\right)^{1 / 2}$ is the associated a posteriori error estimate. It indicates that the meshes and the associated numerical complexity are quasi-optimal: $\mathcal{E}_{k} \approx C N_{k}^{-1 / 3}$ is valid asymptotically.

Figure 6.6 shows the far fields in the direction $(1,0,0)$ for different choices of the PML parameters. Again we observe that the far fields are insensitive to the choices of the PML parameters. 


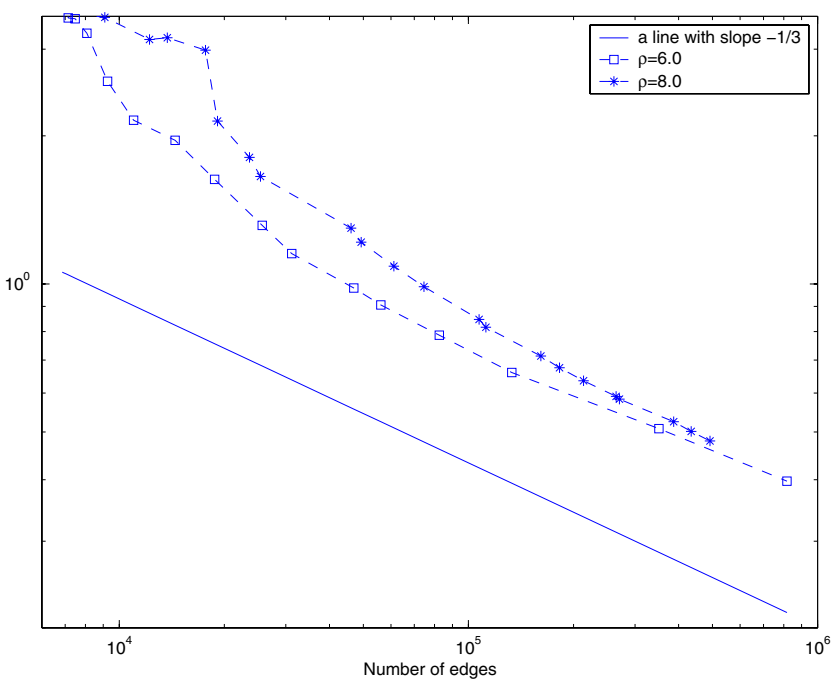

Figure 6.5. The quasi-optimality of the adaptive mesh refinements of the a posteriori error estimator for Example 2.

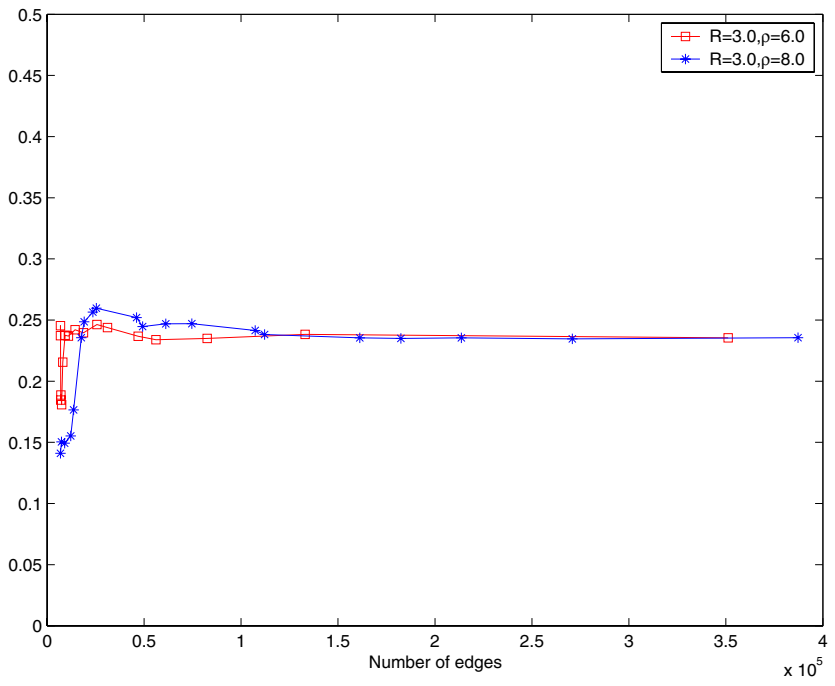

Figure 6.6. The module of the real part of the far fields in the direction $(1,0,0)$ for Example 2.

\section{ACKNOWLEDGEMENTS}

The authors would like to thank Dr. Long Wang and Dr. Weiying Zheng for their help in the computation of this work. The authors are also grateful to the referees for the constructive comment which improved the presentation of the paper. 


\section{REFERENCES}

[1] I. Babuška and A. Aziz, Survey Lectures on Mathematical Foundations of the Finite Element Method, In The Mathematical Foundations of the Finite Element Method with Application to Partial Differential Equations, ed. by A. Aziz, Academic Press, New York, 1973, 5-359. MR0421106 (54:9111)

[2] I. Babuška and C. Rheinboldt, Error estimates for adaptive finite element computations, SIAM J. Numer. Anal. 15 (1978), 736-754. MR0483395 (58:3400)

[3] G. Bao and H.J. Wu, Convergence analysis of the perfectly matched layer problems for timeharmonic Maxwell's equations, SIAM J. Numer. Anal. 43 (2005), 2121-2143. MR2192334 (2007h:65115)

[4] R. Beck, R. Hiptmair, R. Hoppe and B. Wohlmuth, Residual based a posteriori error estimators for eddy current computation, Math. Model. Numer. Anal. 34 (2000), 159-182. MR 1735971 (2000k:65203)

[5] J.-P. Bérénger, A perfectly matched layer for the absorption of electromagnetic waves, J. Comput. Phys. 114 (1994), 185-200. MR1294924 (95e:78002)

[6] M.Sh. Birman and M.Z. Solomyak, $L^{2}$-Theory of the Maxwell operator in arbitary domains, Uspekhi Mat. Nauk, 42 (1987), pp. 61-76 (in Russian); Russian Math. Surveys, 43 (1987), 75-96 (in English). MR933995 (89e:35127)

[7] J.H. Bramble and J.E. Pasciak, Analysis of a finite PML approximation for the three dimensional time-harmonic Maxwell and acoustic scattering problems, Math. Comp. 76 (2007), 597-614. MR2291829

[8] Z. Chen and X.Z. Liu, An adaptive perfectly matched layer technique for time-harmonic scattering problems, SIAM J. Numer. Anal. 43 (2005), 645-671. MR2177884 (2006m:65260)

[9] Z. Chen, L. Wang and W. Zheng, An adaptive multilevel method for time-harmonic Maxwell equations with singularities, SIAM J. Sci. Comput. 29 (2007), 118-138. MR.2285885

[10] Z. Chen and H.J. Wu, An Adaptive Finite Element Method with Perfectly Matched Absorbing Layers for the Wave Scattering by Periodic Structures, SIAM J. Numer. Anal. 41 (2003), 799-826. MR2005183 (2004k:65215)

[11] Ph. Clément, Approximation by finite element functions using local regularization, RAIRO Anal. Numer. 9 (1975), 77-84. MR0400739 (53:4569)

[12] F. Collino and P.B. Monk, The perfectly matched layer in curvilinear coordinates, SIAM J. Sci. Comput. 19 (1998), 2061-2090. MR1638033(99e:78029)

[13] D. Colton and R. Kress, Inverse Acoustic and Electromagnetic Scattering Theory, Springer, 1998. MR.1635980 (99c:35181)

[14] A.B. Dhia, C. Hazard, and S. Lohrengel, A singular field method for the solution of Maxwell's equations in polyhedral domains, SIAM J. Appl. Math. 59 (1999), 2028-2044. MR1709795 (2000g:78032)

[15] T. Hohage, F. Schmidt and L. Zschiedrich, Solving time-harmonic scattering problems based on the pole condition. II: Convergence of the PML method, SIAM J. Math. Anal. 35 (2003), 547-560. MR2048399 (2005f:78003)

[16] M. Lassas and E. Somersalo, On the existence and convergence of the solution of PML equations. Computing 60 (1998), 229-241. MR1621305(99a:65133)

[17] P. Monk, A posteriori error indicators for Maxwell's equations, J. Comp. Appl. Math. 100 (1998), 173-190. MR1659117 (2000k:78020)

[18] P. Monk, Finite Elements Methods for Maxwell Equations, Oxford University Press, 2003. MR2059447 (2005d:65003)

[19] J.C. Nédeléc, Mixed Finite Elements in $\mathbb{R}^{3}$, Numer. Math. 35 (1980), 315-341. MR592160 (81k:65125)

[20] J.C. Nédeléc, Acoustic and Electromagnetic Equations: Integral Representations for Harmonic Problems, Springer, 2001. MR:1822275 (2002c:35003)

[21] A.H. Schatz, An observation concerning Ritz-Galerkin methods with indefinite bilinear forms, Math. Comp. (1974), 959-962. MR0373326 (51:9526)

[22] A. Schmidt and K.G. Siebert, ALBERT: An adaptive hierarchical finite element toolbox, IAM, University of Freiburg, 2000. http://www.mathematik.unifreiburg.de/IAM/Research/projectsdz/albert. 
[23] F.L. Teixeira and W.C. Chew, Advances in the theory of perfectly matched layers, In: W.C. Chew et al., (eds.), Fast and Efficient Algorithms in Computational Electromagnetics, 283-346, Artech House, Boston, 2001.

[24] E. Turkel and A. Yefet, Absorbing PML boundary layers for wave-like equations, Appl. Numer. Math. 27 (1998), 533-557. MR1644675 (99g:78008)

[25] G.N. Watson, A Treatise on The Theory of Bessel Functions, Cambridge, 1922.

Institute of Computational Mathematics, Academy of Mathematics and System Sciences, Chinese Academy of Sciences, Beijing 100080, China

Current address: Department of Mathematics, The Chinese University of Hong Kong, Shatin, N.T., Hong Kong

E-mail address: jqchen@lsec.cc.ac.cn

LSEC, Institute of Computational Mathematics, Academy of Mathematics and System Sciences, Chinese Academy of Sciences, Beijing 100080, China

E-mail address: zmchen@lsec.cc.ac.cn 